AperTO - Archivio Istituzionale Open Access dell'Università di Torino

The time delays influence on the dynamical complexity of algal blooms in the presence of bacteria

This is a pre print version of the following article:

Original Citation:

Availability:

This version is available http://hdl.handle.net/2318/1705911

since 2019-07-09T14:53:06Z

Published version:

DOI:10.1016/j.ecocom.2019.100769

Terms of use:

Open Access

Anyone can freely access the full text of works made available as "Open Access". Works made available under a Creative Commons license can be used according to the terms and conditions of said license. Use of all other works requires consent of the right holder (author or publisher) if not exempted from copyright protection by the applicable law. 


\title{
The time delays influence on the dynamical complexity of algal blooms in the presence of bacteria
}

\author{
Pankaj Kumar Tiwari ${ }^{\mathrm{a}}$, Sudip Samanta ${ }^{\mathrm{b}}$, Francesca Bona $^{\mathrm{c}}$, Ezio Venturino ${ }^{\mathrm{d}}$, Arvind Kumar Misra ${ }^{1 \mathrm{e}}$ \\ ${ }^{a}$ Department of Mathematics, University of Kalyani, Kalyani - 741 235, West Bengal, India \\ ${ }^{b}$ Department of Mathematics, Faculty of Science \& Arts - Rabigh, King Abdulaziz University, Rabigh-25732, Saudi \\ Arabia \\ ${ }^{c}$ DBIOS, University of Turin, via Accademia Albertina 13, 10123 Turin, Italy \\ ${ }^{d}$ Dipartimento di Matematica "Giuseppe Peano", via Carlo Alberto 10, 10123 Torino, Università di Torino, Italy; \\ Member of the INdAM research group GNCS \\ ${ }^{e}$ Department of Mathematics, Institute of Science, Banaras Hindu University, Varanasi 221005, India
}

\begin{abstract}
Bacteria associated with oceanic algal blooms are acknowledged to play important roles in carbon, nitrogen, and sulfur cycling. They influence the climate, mediate primary production, participate in biogeochemical cycles, and maintain ecological balance. A greater insight on the control of the interactions between microalgae and other microorganisms, particularly bacteria, would be helpful in exploring the role of bacteria on algal blooms in lakes. The present study is to investigate the effects of bacteria on the occurrence of algal blooms in lakes. We propose a nonlinear mathematical model by taking into account interactions among nutrients, algae, detritus and bacteria in a lake. We assume that bacteria enhance the growth of algal biomass through remineralization only. Equilibria are analyzed for feasibility and stability, substantiated via numerical simulations. Increase in uptake rate of nutrients by algae and bacteria death rate generates transcritical bifurcations. We perform a global sensitivity analysis to identify the important parameters of the model having a significant impact on the densities of algae and bacteria in the lake. Our findings show that massive algal production might occur in the presence of bacteria, and microalgae-bacteria interactions can be beneficial to the massive production of microalgae. Further, the effect of time delays involved in the bacterial decomposition conversion of detritus into nutrients is studied. Chaotic oscillations may arise via equilibrium destabilization on increasing the values of the time lag. To support chaos occurrence, the Poincaré map is drawn and the Lyapunov exponents are also computed. The findings, critically important for lake restoration, indicate that hypoxia in the lake can be prevented if detritus removal is performed on a regular basis, at time intervals smaller than the critical threshold in the delay with which detritus is decomposed into nutrients.
\end{abstract}

Key words: Mathematical model, Nutrients, Algae-bacteria interactions, Delay, Chaos, Lyapunov exponent, Global sensitivity.

\section{Introduction}

In marine and freshwater ecosystems, phytoplankton transform inorganic carbon of water into organic matter that enters the food web or sinks to the sediments. Approximately one-half of such matter is directly utilized by heterotrophic bacteria, through the water column and at the level of sediments, and newly converted into inorganic compounds: $\mathrm{CO}_{2}$ and nutrients (1). Seasonally in sea and lakes, phytoplankton increase in number determining the so-called blooms, and their requirement of $\mathrm{CO}_{2}$ and nutrients, such as nitrogen and phosphorous, increases consequently (2). Blooms of algae, that

\footnotetext{
${ }^{1}$ Corresponding author. Email: akmisra@bhu.ac.in

Preprint submitted to Ecological Complexity
} 
are sometimes a signal of dangerous eutrophication of waters, are even correlated with a subsequent increase in activity of heterotrophic bacteria that convert algal organic production into bacterial biomass, releasing $\mathrm{CO}_{2}$ and regenerating nutrients associated with algal organic matter (3). With more food available as a consequence of algal blooms, bacteria grow and use up the dissolved oxygen in the water (3). Thus, the content of dissolved oxygen decreases and create hypoxic areas where aquatic life is not longer possible (4). Indeed algal blooms are considered one of the most critical emergence affecting water bodies across the world $(5 ; 6 ; 7 ; 8 ; 9 ; 10)$. Moreover, under eutrophic conditions harmful species of phytoplankton such as toxic cyanobacteria are facilitated and can cause serious adverse effects on animals and humans. They have been reported to kill aquatic life, taint drinking water, contaminate fish and shellfish, making catch inedible $(11 ; 12 ; 13 ; 14)$. Eutrophication negatively affects local economies and ecosystem services such as fishing, tourism, recreation and real estate $(15 ; 16)$.

Phytoplankton and bacterioplankton interact continuously: their communities are closely interrelated in terms of abundance and functions (17). Moreover, heterotrophic bacteria satisfy their carbon requirement not only by using phytoplankton derived matter but also by degrading all organic compounds released into the aquatic ecosystems by human activities, and therefore nutrients produced by bacteria from sewage are another resource supporting the algal growth (3). Bacteria can also compete with planktonic algae for the same inorganic resources, principally at the earliest and terminal stages of a bloom when nutrients are become limiting (3). Clearly, phytoplankton blooms create environmental conditions favouring the increase in the bacterial growth and production (18). Bacterial and algal biomasses are generally correlated at the height of the bloom, while algae prevail during the earliest stage when nutrients are scarce. Bacteria remain abundant when blooms decline as they continue to utilize the organic substances released by dying and dead algae (19). Two distinct phases can be recognized in the release of organic compounds by phytoplankton. In the earliest stages of a bloom, algae release soluble, low molecular weight compounds which can act as chemical attractants for bacteria. In the declining stage, phytoplankton release higher molecular weight macromolecules such as polysaccharides, proteins and lipids, that can be assimilated by bacteria.

Heterotrophic bacteria assimilate and remineralize the organic matter firstly by converting it to the dissolved phase thanks to the action of their extracellular and cell-surface enzymes. Then such dissolved molecules are readily transported across the cell wall and transformed into bacterial biomass. In lab cultures flavobacteria and roseobacter have been seen to directly attack viable algal cells (20). However, their interactions with phytoplankton are essentially mutualistic, as they provide algae with nutrients, vitamins and growth promoting factors, such as the auxins. But at the end of a bloom when nutrients become limiting, symbiosis can be converted in competition as roseobacteria in particular produce algicidal compounds favouring the decline of algae community (21). The interactions between these two communities are areas of recent studies $(22 ; 23)$, which shed light on the complex role of heterotrophic bacteria in algal growth and survival. They not only decompose organic matter allowing the nutrient recycling, but also promote algal growth by complex communication mechanisms and nutrient exchange (24). Indeed not only macro-nutrients such as nitrogen, phosphorus and carbon are exchanged between them but also micro-nutrients such as vitamins and other growth-promoting molecules $(25 ; 26)$.

Several studies have been conducted to explore the role of nutrients on the bloom of algae in lakes/ponds $(6 ; 7 ; 8 ; 9 ; 10)$. Deeper insights into the role of heterotrophic bacteria may have important applications in the strategies to control algal blooms in aquatic ecosystems. Moreover, nowadays algaebacteria interactions are considered as promising in biotechnology, as recent studies highlighted the positive role of bacteria for massive algal cultivation for biotechnological applications $(20 ; 22 ; 23 ; 27 ; 28 ; 29)$. However, in $(6 ; 7 ; 8 ; 9 ; 10)$ models on the recycling of detritus into nutrients do not explicitly account for the role of bacteria. It is well documented that bacteria play a potentially important role in structuring 
algal species (30). Thus, the possible effects of bacteria on the population dynamics of algal species must be considered in the modelling process. Therefore, in this paper, our aim is to assess the effect of algae-bacteria interactions on the dynamics of algal population.

In many processes of real phenomena, the present dynamics of the state variables depends not only on the present state of the processes but also on their past history (31). These processes are generally modeled by time delays which are known to trigger instabilities $(32 ; 33)$. The potential effects of nutrient recycling on the ecosystem has been studied $(6 ; 34 ; 35)$, but it is not an instantaneous process. The time needed by bacteria to transform the mass of dead algae into nutrients has not been explicitly taken into account, in general, but it should, in order to represent a complete nutrient cycle (36). Plankton-nutrient interaction models with both instantaneous and delayed nutrient recycling show that the stability of the positive equilibrium is lost after the input rate of nutrient crosses a threshold value and oscillations emerges through Hopf bifurcation (37). A planktonic resource-consumer model exhibiting a temporal delay in the formation of nutrients from the dead plankton is presented in (38), where the nutrient inflows at a constant rate. The system may destabilize due to this temporal lag. Other similar investigations involve e.g. delays on nutrient cycling in phytoplankton-zooplankton interactions in an estuarine system (39), discrete time delays in the conversion of detritus into nutrients, for which upon crossing a threshold, the system may switch finitely many times back and forth from stability to instability, but eventually becoming unstable (40). Control measures have therefore been considered in (41): the presence of two time delays may produce a stabilizing effect on the system dynamics. In view of these considerations, here we study the effects of time delays on the algae-bacteria interactions, due to the time lags needed for the bacterial conversion of detritus into nutrients $(36 ; 38 ; 40 ; 41)$. The possibility of Hopf-bifurcation is explored by taking the time delay as a bifurcation parameter.

The rest of the paper is organized as follows: in the next section, we formulate the model. In Section 3, we study the dynamics of nutrients-algae system only, while in Section 4, we include detritus also, then in Section 5, we consider the input rate of nutrients from external sources as zero. In Section 6, we analyze the full model, and in Section 7, we show that system's equilibria are related via transcritical bifurcations. In Section 8, we consider the modified model to account for time delays in the decomposition and regeneration processes. Hopf-bifurcation at the coexistence equilibrium are studied in Section 9. In Section 10, we validate our analytical findings through numerical simulations. A global sensitivity analysis is performed to identify the most influential model parameters that have significant impact on algae and bacteria. We conclude with a wrap-up discussion.

\section{The mathematical model}

Currently there are more than 500 hypoxic systems covering over 2,40,000 $\mathrm{km}^{2}$ around the globe (42). In China, several lakes such as lake Chaohu, Lake Taihu and Dianchi had been examined and data showed that they were in eutrophication (43). Algae, heterotrophic bacteria and Archea are the primary producers and decomposers, respectively, making them the structural pillars of the ecosystem and its foremost functional entities. Pseudomonas diminuta and Pseudomonas vesicularis, two obligate aerobes, stimulated the growth of the green microalgae Scenedesmus bicellularis and Chlorellu sp., without releasing any growth-promoting substance (44). Indeed, under controlled conditions with a limited concentration of inorganic carbon, Mouget et al. (44) found that the presence of $P$. diminuta stimulated the photosynthetic activity of $S$. bicellularis. These authors suggest that aerobic bacteria can enhance algal productivity by attenuating the photosynthetic oxygen tension around algal cells. Moreover, bacteria may become an important source of carbon from algal growth, especially under conditions of $\mathrm{CO}_{2}$ limitation (45). Finally, they can promote algal growth by releasing vitamins and other growth factors or by modifying some physico-chemical property of the aquatic environment such as the $\mathrm{pH}(46)$. In 
most cases the association between algae and bacteria leads to mutual interactions (47). Indeed algae provide oxygen and organic molecules to support bacteria growth, despite in some phases of algal bloom their interaction may include resource (e.g. nutrients) competition. However, most types of interactions between algae and bacteria in the planktonic zone are scantily studied. The ones studied often reflect the importance of undertaking such studies (3). Here, we consider the growth of algae due to bacteria only via nutrient regeneration on decomposition of organic matter, no other mode is considered $(3 ; 44)$.

Consider a lake which is being eutrophied due to overgrowth of algae and other biological species caused by discharge of nutrients from domestic drainage as well as from water run off, etc., and also from nutrients formed from detritus upon bacterial decomposition. We consider four dependent variables in the lake, namely; concentration of nutrients (nitrogen, phosphorus, etc.), $N(\mu g / L)$, density of algal populations, $A(\mu g / L)$, density of detritus (formed due to death of algae), $D(\mu g / L)$, and density of bacteria, $B(\mu \mathrm{g} / L)$, at any time $t>0$. Algae in lakes consist of diverse assemblage of all major taxonomic groups. Many of these forms have different physiological requirements and differ in their response to light, temperature and concentration of nutrients. Here, we do not consider the effects of light and temperature on the algal growth and assume that the algal population fully depends on the availability of nutrients in the lake. It is assumed that nutrients are continuously discharged into the lake from different sources (domestic drainage, water run off from agricultural fields, etc.) at a constant rate, being naturally depleted and utilized by algae. The utilization rate of nutrients by algae is assumed in the form of a Holling type-II term and the growth rate of algal populations is proportional to the same interaction term. Algae are removed by natural death, higher predation and intraspecific competition and then are turned into detritus which either naturally depletes or is decomposed by the bacterial pool, this being expressed once more via a Holling type-II function. Depletion of detritus occurs due to biochemical processes performed by anaerobic and aerobic bacteria that convert it into nutrients. Conversion by anaerobic bacteria does not need dissolved oxygen (DO), but the latter is required in the aerobic processes for nutrient degradation. This results in the depletion of DO level in the water body. However, in this paper the bacteria decomposition process is considered without explicitly writing an equation for DO level. After decomposition by bacteria, detritus are recycled into nutrients (remineralization) and the bacterial population increases due to this conversion process. Further, the density of bacteria increases in the aquatic environment due to other types of detritus, different from the algal one. Bacteria natural mortality is taken into account as well as due to other causes, such as enzymatic attack, temperature variations, UV radiation, photo-oxidation, etc. (48).

Algal bloom has pushed the problem of the quality and quantity of water available for consumption to alarming levels, so that nowadays the existence of aquatic life is threatened $(10 ; 49 ; 50)$. Large amounts of decaying biological materials in the water bodies lead to oxygen depletion, and this results in fish population collapse, as the oxygen saturation level has an effect on growth and feed conversion ratios of fish. Due to low levels of DO, frequently associated with phenomena such as heat waves, cloudy weather, organic overload, algae die-offs, or heavy thunderstorms (51), the whole aquatic ecosystem is significantly affected and survival of the aquatic species is threatened. Incorporating fish populations and DO levels in the model formulation would be more realistic, but would by far increase the complexity of the model and therefore is not considered here. Other related studies have in part considered these issues, see $(52 ; 53)$.

The schematic diagram for the interactions among nutrients, algae, detritus and bacteria is depicted in Fig. 1. The resulting system, in which all the parameters are assumed to be positive and constant, 


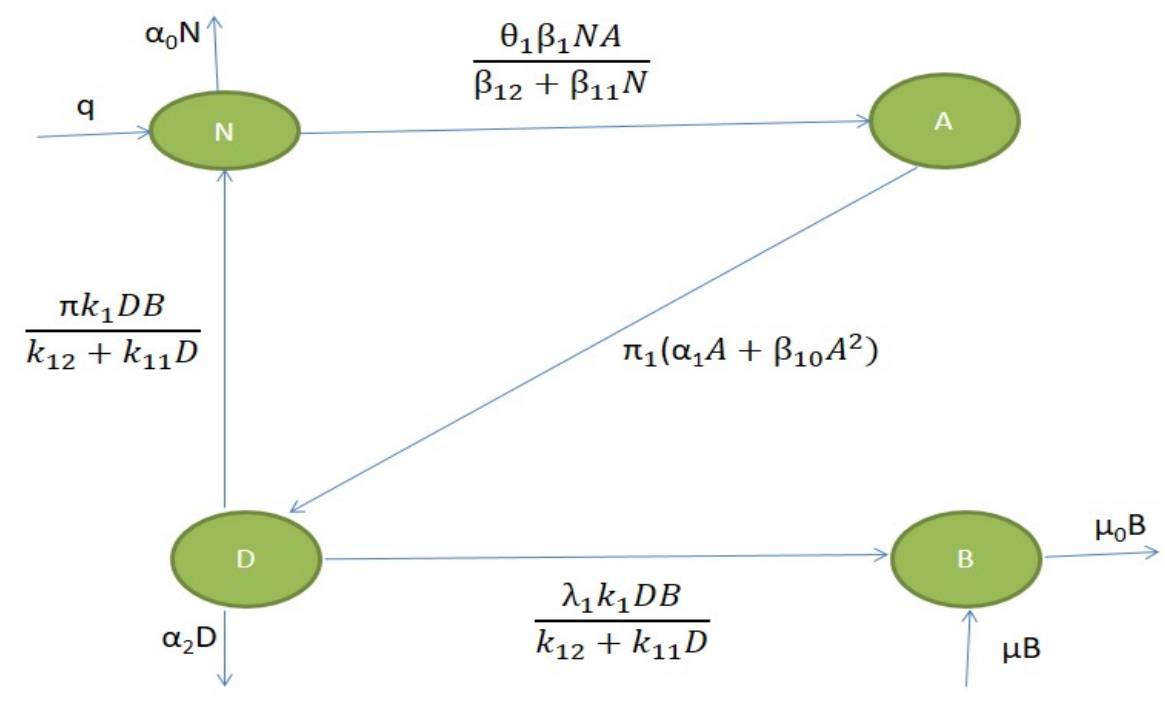

Figure 1: Schematic diagram for the interactions among nutrients, algae, detritus and bacteria.

reads:

$$
\begin{aligned}
\frac{d N}{d t} & =q-\alpha_{0} N-\frac{\beta_{1} N A}{\beta_{12}+\beta_{11} N}+\frac{\pi k_{1} D B}{k_{12}+k_{11} D}, \\
\frac{d A}{d t} & =\frac{\theta_{1} \beta_{1} N A}{\beta_{12}+\beta_{11} N}-\alpha_{1} A-\beta_{10} A^{2}, \\
\frac{d D}{d t} & =\pi_{1}\left(\alpha_{1} A+\beta_{10} A^{2}\right)-\alpha_{2} D-\frac{k_{1} D B}{k_{12}+k_{11} D} \\
\frac{d B}{d t} & =\mu B-\mu_{0} B+\frac{\lambda_{1} k_{1} D B}{k_{12}+k_{11} D} .
\end{aligned}
$$

The biological meaning of the parameters involved in the system (2.1) and their values used for numerical simulations are given in Table 1 . The constant $\pi_{1}$ represents the conversion of dead algae into detritus due to natural death, higher predation and intraspecies competition $(6 ; 54)$. For the biological soundness (i.e. boundedness of the trajectories) of the model, the bacteria natural death rate is assumed to be higher than its growth rate due to detritus types other than algal one, i.e., $\mu_{0}-\mu$ is positive. Also, the following two conditions must hold

$$
\begin{aligned}
\theta_{1} \beta_{1}-\beta_{11} \alpha_{1} & >0, \\
\lambda_{1} k_{1}-k_{11}\left(\mu_{0}-\mu\right) & >0 .
\end{aligned}
$$

Biologically, to satisfy condition (2.2), the total benefit to the algal biomass on consumption of nutrients must exceed the natural death rate of algae. If this condition fails, the growth rate of algae becomes negative and thus as time increases algae disappear. Similarly, condition (2.3) means that the bacteria 
population growth caused by detritus decomposition must be greater than the difference between the natural death rate of bacteria and the bacterial population growth due to other types of detritus. If the condition does not hold, then the bacteria population declines, and eventually vanishes as time increases. To have a meaningful model, the algal and bacterial populations must thrive, thus the conditions (2.2) and (2.3) must hold.

Table 1: Parameter values (hypothetical) in the system (2.1)

\begin{tabular}{|c|c|c|c|c|}
\hline Names & Description & Unit & Value & Reference \\
\hline$q$ & $\begin{array}{l}\text { Input rate of nutrients to the lake from domestic } \\
\text { drainage and water run off from agricultural fields }\end{array}$ & $\mu \mathrm{g} / \mathrm{L} /$ day & 0.05 & $(8)$ \\
\hline$\alpha_{0}$ & $\begin{array}{l}\text { Per capita loss rate of nutrient due to sinking } \\
\text { from the epilimnion down to the hypolimnion and thus } \\
\text { making these nutrients unavailable for algae uptake }\end{array}$ & 1/day & 0.01 & $(10)$ \\
\hline$\beta_{1}$ & Maximum uptake rate of nutrients by algae & 1/day & 0.85 & \\
\hline$\beta_{12}$ & Half saturation constant & $\mu \mathrm{g} / \mathrm{L} /$ day & 1 & \\
\hline$\beta_{11}$ & Proportionality constant & - & 10 & \\
\hline$\pi$ & Remineralization of detritus into nutrients & - & 0.3 & \\
\hline$k_{1}$ & Maximum decomposition rate of detritus by bacteria & 1/day & 0.5 & \\
\hline$k_{12}$ & Half saturation constant & $\mu \mathrm{g} / \mathrm{L} /$ day & 1 & \\
\hline$k_{11}$ & Proportionality constant & - & 0.3 & \\
\hline$\theta_{1}$ & Algal growth due to nutrients uptake & - & 5 & \\
\hline$\alpha_{1}$ & Natural mortality and higher predation of algae & 1/day & $1 / 3$ & \\
\hline$\beta_{10}$ & Algal mortality due to intraspecific competition & $\mathrm{L} / \mu \mathrm{g} /$ day & 0.01 & \\
\hline$\pi_{1}$ & Algal conversion into detritus & - & 0.2 & \\
\hline$\alpha_{2}$ & $\begin{array}{l}\text { Sinking rate of detritus to the bottom of the lake, } \\
\text { being buried into the sediments at the lake bottom }\end{array}$ & 1/day & 0.006 & \\
\hline$\mu$ & Bacterial growth due to other types of detritus & 1/day & 1.85 & \\
\hline$\mu_{0}$ & Natural death rate of bacteria & 1/day & 2 & \\
\hline$\lambda_{1}$ & Bacterial growth due to detritus & - & 0.5 & \\
\hline
\end{tabular}

\section{Ecosystem with nutrients and algae only}

Considering a special case of system (2.1) where only the dynamics of nutrients and algae is taken into account, then we have the following subsystem

$$
\begin{aligned}
\frac{d N}{d t} & =q-\alpha_{0} N-\frac{\beta_{1} N A}{\beta_{12}+\beta_{11} N}, \\
\frac{d A}{d t} & =\frac{\theta_{1} \beta_{1} N A}{\beta_{12}+\beta_{11} N}-\alpha_{1} A-\beta_{10} A^{2} .
\end{aligned}
$$

\subsection{Boundedness}

The feasible region for system (3.1) is given in the following lemma.

Lemma 3.1. The region of attraction for all solutions of system (1) initiating in the positive quadrant is given by (55):

$$
\Omega_{1}=\left\{(N, A) \in \mathbb{R}_{+}^{2}: 0<N+P \leq q / \delta_{m}\right\},
$$

where $\delta_{m}=\min \left\{\alpha_{0}, \alpha_{1}\right\}$. The region $\Omega_{1}$ is closed and bounded in the positive quadrant of the two dimensional plane. Consequently, the system (3.1) is dissipative and any solution is defined for $t \geq 0$. 


\subsection{Equilibria}

System (3.1) has two feasible equilibria: (i) The algae-free equilibrium $\bar{E}_{0}=\left(q \alpha_{0}^{-1}, 0\right)$, always feasible, and (ii) The interior equilibrium $\bar{E}^{*}=\left(\bar{N}^{*}, \bar{A}^{*}\right)$, where $\bar{N}^{*}=\frac{\beta_{12}\left(\alpha_{1}+\beta_{10} \bar{A}^{*}\right)}{\left(\theta_{1} \beta_{1}-\beta_{11} \alpha_{1}\right)-\beta_{11} \beta_{10} \bar{A}^{*}}$ and $\bar{A}^{*}$ is a positive root of the following equation:

$$
f(A)=\left[q-\frac{A\left(\alpha_{1}+\beta_{10} A\right)}{\theta}\right]\left[\left(\theta_{1} \beta_{1}-\beta_{11} \alpha_{1}\right)-\beta_{11} \beta_{10} A\right]-\beta_{10} \alpha_{0}\left(\alpha_{1}+\beta_{10} A\right) .
$$

From equation (3.2), we note the following

1. $f(0)=q\left(\theta_{1} \beta_{1}-\beta_{11} \alpha_{1}\right)-\beta_{12} \alpha_{1} \alpha_{0}$, which is positive provided

$$
q\left(\theta_{1} \beta_{1}-\beta_{11} \alpha_{1}\right)>\beta_{12} \alpha_{1} \alpha_{0} .
$$

2. $f(\bar{A})<0$, where $\bar{A}=\frac{\theta_{1} \beta_{1}-\beta_{11} \alpha_{1}}{\beta_{11} \beta_{10}}$.

3. $f^{\prime}(A)<0, \forall A \in(0, \bar{A})$.

Thus, equation (3.2) has exactly one positive root in the interval $(0, \bar{A})$ and the interior equilibrium $\bar{E}^{*}$ is feasible provided condition (3.3) holds.

\subsection{Stability}

3.3.1. Local stability

The Jacobian of system (3.1) is

$$
\bar{J}=\left(\begin{array}{cc}
-\left(\alpha_{0}+\frac{\beta_{1} \beta_{12} A}{\left(\beta_{12}+\beta_{11} N\right)^{2}}\right) & -\frac{\beta_{1} N}{\beta_{12}+\beta_{11} N} \\
\frac{\theta_{1} \beta_{1} \beta_{12} A}{\left(\beta_{12}+\beta_{11} N\right)^{2}} & \frac{\theta_{1} \beta_{1} N}{\beta_{12}+\beta_{11} N}-\alpha_{1}-2 \beta_{10} A
\end{array}\right) .
$$

At equilibrium $\bar{E}_{0}$, the eigenvalues are

$$
-\alpha_{0}, \frac{\theta_{1} \beta_{1} q}{\beta_{12} \alpha_{0}+\beta_{11} q}-\alpha_{1} .
$$

One eigenvalue is negative while the other is negative if condition (3.3) does not hold. Thus, the equilibrium $\bar{E}_{0}$ is related via transcritical bifurcation to the equilibrium $\bar{E}^{*}$.

The characteristic equation at the equilibrium $\bar{E}^{*}$ is

$$
\begin{aligned}
\lambda^{2}+\lambda\left(\alpha_{0}+\right. & \left.\beta_{10} \bar{A}^{*}+\frac{\beta_{1} \beta_{12} \bar{A}^{*}}{\left(\beta_{12}+\beta_{11} \bar{N}^{*}\right)^{2}}\right)+\beta_{10} \bar{A}^{*}\left(\alpha_{0}+\frac{\beta_{1} \beta_{12} \bar{A}^{*}}{\left(\beta_{12}+\beta_{11} \bar{N}^{*}\right)^{2}}\right) \\
& +\frac{\theta_{1} \beta_{1} \beta_{12} \bar{A}^{*}}{\left(\beta_{12}+\beta_{11} \bar{N}^{*}\right)^{2}} \frac{\beta_{1} \bar{N}^{*}}{\beta_{12}+\beta_{11} \bar{N}^{*}}=0 .
\end{aligned}
$$

Since the linear and constant terms are positive, in view of Routh-Hurwitz criterion, the roots are either negative or have negative real parts. Thus, the equilibrium $\bar{E}^{*}$ is unconditionally stable.

The results can be summarized in the following theorem.

Theorem 3.1. $\quad$ 1. The algae-free equilibrium $\bar{E}_{0}$ is related to the interior equilibrium $\bar{E}^{*}$ via a transcritical bifurcation.

2. The interior equilibrium $\bar{E}^{*}$, if feasible, is unconditionally locally asymptotically stable. 


\subsubsection{Global stability}

As far as global stability of the equilibrium $\bar{E}^{*}$ is concerned, we have the following result.

Theorem 3.2. The coexistence equilibrium $\bar{E}^{*}$, if feasible, is globally asymptotically stable inside the region of attraction $\Omega_{1}$ provided the following condition holds:

$$
\left[\frac{\beta_{1} \beta_{11} q}{\left(\beta_{12} \delta_{m}+\beta_{11} q\right)\left(\beta_{12}+\beta_{11} \bar{N}^{*}\right)}\right]^{2} \bar{N}^{*}<\frac{4 \beta_{10} \alpha_{0}}{\theta_{1}} .
$$

Proof. We consider the following as a Lyapunov function candidate

$$
U=\frac{1}{2}\left(N-\bar{N}^{*}\right)^{2}+m_{1}\left(A-\bar{A}^{*}-\bar{A}^{*} \ln \frac{A}{\bar{A}^{*}}\right),
$$

226

227 of the system (3.1) is

$$
\begin{aligned}
\dot{U}=- & {\left[\alpha_{0}+\frac{\beta_{1} \beta_{12} A}{\left(\beta_{12}+\beta_{11} N\right)\left(\beta_{12}+\beta_{11} \bar{N}^{*}\right)}\right]\left(N-\bar{N}^{*}\right)^{2}-m_{1} \beta_{10}\left(A-\bar{A}^{*}\right)^{2} } \\
& +\left[\frac{m_{1} \theta_{1} \beta_{1} \beta_{12}}{\left(\beta_{12}+\beta_{11} N\right)\left(\beta_{12}+\beta_{11} \bar{N}^{*}\right)}-\frac{\beta_{1} \bar{N}^{*}}{\beta_{12}+\beta_{11} \bar{N}^{*}}\right]\left(N-\bar{N}^{*}\right)\left(A-\bar{A}^{*}\right) .
\end{aligned}
$$

Set $m_{1}=\bar{N}^{*} / \theta_{1}, \dot{U}$ is negative definite inside $\Omega_{1}$ provided condition (3.4) holds.

\section{Ecosystem behavior in the absence of bacteria}

If we consider the case when no bacteria is present in the aquatic environment, then system (2.1) takes the following form

$$
\begin{aligned}
\frac{d N}{d t} & =q-\alpha_{0} N-\frac{\beta_{1} N A}{\beta_{12}+\beta_{11} N} \\
\frac{d A}{d t} & =\frac{\theta_{1} \beta_{1} N A}{\beta_{12}+\beta_{11} N}-\alpha_{1} A-\beta_{10} A^{2} \\
\frac{d D}{d t} & =\pi_{1}\left(\alpha_{1} A+\beta_{10} A^{2}\right)-\alpha_{2} D
\end{aligned}
$$

\subsection{Boundedness}

The feasible region for system (4.1) is given in the following lemma.

Lemma 4.1. The region of attraction for all solutions of system (4.1) initiating in the positive octant is given by (55):

$$
\Omega_{2}=\left\{(N, A, D) \in \mathbb{R}_{+}^{3}: 0<N+P+D \leq q / \delta_{n}\right\}
$$

where $\delta_{n}=\min \left\{\alpha_{0},(1-\pi) \alpha_{1}, \alpha_{2}\right\}$. The region $\Omega_{2}$ is closed and bounded in the positive cone of the three dimensional space. Consequently, the system (4.1) is dissipative and any solution is defined for $t \geq 0$. 


\subsection{Equilibria}

System (4.1) has two feasible equilibria: (i) The algae-detritus-free equilibrium $\widehat{E}_{0}=\left(q \alpha_{0}^{-1}, 0,0\right)$, always feasible, and (ii) The interior equilibrium $\widehat{E}^{*}=\left(\widehat{N}^{*}, \widehat{A}^{*}, \widehat{D}^{*}\right)$, where

$$
\widehat{N}^{*}=\frac{\beta_{12}\left(\alpha_{1}+\beta_{10} \widehat{A}^{*}\right)}{\left(\theta_{1} \beta_{1}-\beta_{11} \alpha_{1}\right)-\beta_{11} \beta_{10} \widehat{A}^{*}}, \widehat{D}^{*}=\frac{\pi_{1} \widehat{A}^{*}\left(\alpha_{1}+\beta_{10} \widehat{A}^{*}\right)}{\alpha_{2}}
$$

and $\widehat{A}^{*}$ is a positive root of the following equation:

$$
g(A)=\left[q-\frac{A\left(\alpha_{1}+\beta_{10} A\right)}{\theta}\right]\left[\left(\theta_{1} \beta_{1}-\beta_{11} \alpha_{1}\right)-\beta_{11} \beta_{10} A\right]-\beta_{10} \alpha_{0}\left(\alpha_{1}+\beta_{10} A\right) .
$$

From (3.2), we have

1. $g(0)=q\left(\theta_{1} \beta_{1}-\beta_{11} \alpha_{1}\right)-\beta_{12} \alpha_{1} \alpha_{0}$, which is positive provided

$$
q\left(\theta_{1} \beta_{1}-\beta_{11} \alpha_{1}\right)>\beta_{12} \alpha_{1} \alpha_{0} .
$$

2. $g(\bar{A})<0$, where $\bar{A}=\frac{\theta_{1} \beta_{1}-\beta_{11} \alpha_{1}}{\beta_{11} \beta_{10}}$.

3. $g^{\prime}(A)<0, \forall A \in(0, \bar{A})$.

Thus, the equation (4.2) has exactly one positive root in the interval $(0, \bar{A})$. Thus, the interior equilibrium $\widehat{E}^{*}$ is feasible provided condition (4.3) holds.

\subsection{Stability}

4.3.1. Local stability

The Jacobian of system (4.1) is

$$
\bar{J}=\left(\begin{array}{ccc}
-\left(\alpha_{0}+\frac{\beta_{1} \beta_{12} A}{\left(\beta_{12}+\beta_{11} N\right)^{2}}\right) & -\frac{\beta_{1} N}{\beta_{12}+\beta_{11} N} & 0 \\
\frac{\theta_{1} \beta_{1} \beta_{12} A}{\left(\beta_{12}+\beta_{11} N\right)^{2}} & \frac{\theta_{1} \beta_{1} N}{\beta_{12}+\beta_{11} N}-\alpha_{1}-2 \beta_{10} A & 0 \\
0 & \pi_{1}\left(\alpha_{1}+2 \beta_{10} A\right) & -\alpha_{2}
\end{array}\right) .
$$

At equilibrium $\widehat{E}_{0}$, the eigenvalues are

$$
-\alpha_{0}, \frac{\theta_{1} \beta_{1} q}{\beta_{12} \alpha_{0}+\beta_{11} q}-\alpha_{1},-\alpha_{2} .
$$
negative or have negative real parts. Thus, the equilibrium $\widehat{E}^{*}$ is always stable.

Now, we summarize the results in the following theorem.

Theorem 4.1. $\quad$ 1. The algae-free equilibrium $\widehat{E}_{0}$ is related to the interior equilibrium $\widehat{E}^{*}$ via a transcritical bifurcation.

2. The interior equilibrium $\widehat{E}^{*}$, if feasible, is always locally asymptotically stable. 


\subsubsection{Global stability}

For global stability of equilibrium $\widehat{E}^{*}$, we have the following result.

Theorem 4.2. The interior equilibrium $\widehat{E}^{*}$, if feasible, is globally asymptotically stable inside the region of attraction $\Omega_{2}$ provided the following conditions hold

$$
\begin{aligned}
& {\left[\frac{\beta_{1} \beta_{11} q}{\left(\beta_{12} \delta_{n}+\beta_{11} q\right)\left(\beta_{12}+\beta_{11} \widehat{N}^{*}\right)}\right]^{2} \widehat{N}^{*}<\frac{2 \beta_{10} \alpha_{0}}{\theta_{1}},} \\
& \pi_{1}^{2}\left[\alpha_{1}+\beta_{10}\left(q / \delta_{n}+\widehat{A}^{*}\right)\right]^{2}<\frac{2 \beta_{10} \alpha_{2} \widehat{N}^{*}}{\theta_{1}} .
\end{aligned}
$$

Proof. We consider the following as a Lyapunov function candidate

$$
V=\frac{1}{2}\left(N-\widehat{N}^{*}\right)^{2}+m_{1}\left(A-\widehat{A}^{*}-\widehat{A}^{*} \ln \frac{A}{\widehat{A}^{*}}\right)+\frac{m_{2}}{2}\left(D-\widehat{D}^{*}\right)^{2},
$$

where $m_{1}$ and $m_{2}$ are positive constants to be determined. The time derivative of $V$ along the solutions trajectory of the system (4.1) is

$$
\begin{aligned}
\dot{V}=- & {\left[\alpha_{0}+\frac{\beta_{1} \beta_{12} A}{\left(\beta_{12}+\beta_{11} N\right)\left(\beta_{12}+\beta_{11} \widehat{N}^{*}\right)}\right]\left(N-\widehat{N}^{*}\right)^{2}-m_{1} \beta_{10}\left(A-\widehat{A}^{*}\right)^{2}-m_{2} \alpha_{2}\left(D-\widehat{D}^{*}\right)^{2} } \\
& +\left[\frac{m_{1} \theta_{1} \beta_{1} \beta_{12}}{\left(\beta_{12}+\beta_{11} N\right)\left(\beta_{12}+\beta_{11} \widehat{N}^{*}\right)}-\frac{\beta_{1} \widehat{N}^{*}}{\beta_{12}+\beta_{11} \widehat{N}^{*}}\right]\left(N-\widehat{N}^{*}\right)\left(A-\widehat{A}^{*}\right) \\
& +m_{2} \pi_{1}\left[\alpha_{1}+\beta_{10}\left(A+\widehat{A}^{*}\right)\right]\left(A-\widehat{A}^{*}\right)\left(D-\widehat{D}^{*}\right) .
\end{aligned}
$$

Setting $m_{1}=\bar{N}^{*} / \theta_{1}$ and $m_{2}=1, \dot{V}$ is negative definite inside $\Omega_{2}$ provided conditions (4.4) and (4.5) are satisfied.

\section{No nutrients input from external sources}

In all the previous cases, as well as in the full model to be analyzed in the next section, the equilibria always show a nonnegative value for the nutrients. This is evident mathematically and biologically, as their continuous input from external sources prevents them to disappear. To better focus on this situation, we now examine the case in which this inflow is prevented. It turns out anyway, that no nutrient-free equilibrium can be achieved as it will be apparent from the analysis below. The biological reason is that nutrients are replenished by the detritus decomposed by bacteria, and only if one of these two population vanishes, they are also doomed. But in turn, the disappearance of either detritus or bacteria is ultimately related to the total ecosystem collapse or no nutrients production: for no detritus to be generated, the algae must vanish; if bacteria are absent, no conversion to nutrients occurs, and detritus can only sink to the bottom layers of the lake. Assume therefore that nutrients present in the lake are only due to recycling of detritus upon bacterial decomposition. In this particular case, we set $q=0$, and hence equation (2.1) reduces to

$$
\begin{aligned}
\frac{d N}{d t} & =\frac{\pi k_{1} D B}{k_{12}+k_{11} D}-\alpha_{0} N-\frac{\beta_{1} N A}{\beta_{12}+\beta_{11} N}, \\
\frac{d A}{d t} & =\frac{\theta_{1} \beta_{1} N A}{\beta_{12}+\beta_{11} N}-\alpha_{1} A-\beta_{10} A^{2}, \\
\frac{d D}{d t} & =\pi_{1}\left(\alpha_{1} A+\beta_{10} A^{2}\right)-\alpha_{2} D-\frac{k_{1} D B}{k_{12}+k_{11} D} \\
\frac{d B}{d t} & =\mu B-\mu_{0} B+\frac{\lambda_{1} k_{1} D B}{k_{12}+k_{11} D} .
\end{aligned}
$$




\subsection{Equilibria}

System (5.1) has two feasible equilibria:

1. The origin $\widetilde{E}_{0}=(0,0,0,0)$, which is always feasible.

2. The interior equilibrium $\widetilde{E}^{*}=\left(\widetilde{N}^{*}, \widetilde{A}^{*}, \widetilde{D}^{*}, \widetilde{B}^{*}\right)$, with

$$
\begin{gathered}
\widetilde{N}^{*}=\frac{\beta_{12}\left(\alpha_{1}+\beta_{10} \widetilde{A}^{*}\right)}{\left(\theta_{1} \beta_{1}-\beta_{11} \alpha_{1}\right)-\beta_{11} \beta_{10} \widetilde{A}^{*}}, \quad \widetilde{D}^{*}=\frac{k_{12}\left(\mu_{0}-\mu\right)}{\lambda_{1} k_{1}-k_{11}\left(\mu_{0}-\mu\right)}, \\
\widetilde{B}^{*}=\frac{1}{\mu_{0}-\mu}\left[\pi_{1} \widetilde{A}^{*}\left(\alpha_{1}+\beta_{10} \widetilde{A}^{*}\right)-\frac{k_{12} \alpha_{2}\left(\mu_{0}-\mu\right)}{\lambda_{1} k_{1}-k_{11}\left(\mu_{0}-\mu\right)}\right]
\end{gathered}
$$

and $\widetilde{A}^{*}$ is a positive root of the equation

$$
c_{3} A^{3}+c_{2} A^{2}+c_{1} A+c_{0}=0,
$$

where

$$
\begin{aligned}
& c_{3}=\beta_{10}^{2} \beta_{11}\left(1-\pi \pi_{1} \theta_{1}\right)\left\{\lambda_{1} k_{1}-k_{11}\left(\mu_{0}-\mu\right)\right\}, \\
& c_{2}=-\beta_{10}\left(1-\pi \pi_{1} \theta_{1}\right)\left\{\lambda_{1} k_{1}-k_{11}\left(\mu_{0}-\mu\right)\right\}\left(\theta_{1} \beta_{1}-2 \beta_{11} \alpha_{1}\right), \\
& c_{1}=-k_{12} \alpha_{2} \theta_{1} \beta_{11} \beta_{10}+\left\{\lambda_{1} k_{1}-k_{11}\left(\mu_{0}-\mu\right)\right\}\left[\beta_{12} \beta_{10} \theta_{1} \alpha_{0}+\alpha_{1}\left(1-\pi \pi_{1} \theta_{1}\right)\left(\theta_{1} \beta_{1}-\beta_{11} \alpha_{1}\right)\right], \\
& c_{0}=-\theta_{1}\left[k_{12} \alpha_{2}\left(\mu_{0}-\mu\right)\left(\theta_{1} \beta_{1}-\beta_{11} \alpha_{1}\right)+\beta_{12} \alpha_{1} \alpha_{0}\left\{\lambda_{1} k_{1}-k_{11}\left(\mu_{0}-\mu\right)\right\}\right] .
\end{aligned}
$$

In view of assumptions (2.2) and (2.3), equation (5.2) has exactly one positive if the following condition holds:

$$
\theta_{1} \beta_{1}-2 \beta_{11} \alpha_{1}>0
$$

Clearly, $\widetilde{D}^{*}$ is positive in view of assumption $(2.3)$, while $\widetilde{N}^{*}$ and $\widetilde{B}^{*}$ are positive if respectively the following condition hold

$$
\left(\theta_{1} \beta_{1}-\beta_{11} \alpha_{1}\right)-\beta_{11} \beta_{10} \widetilde{A}^{*}>0, \pi_{1} \widetilde{A}^{*}\left(\alpha_{1}+\beta_{10} \widetilde{A}^{*}\right)-\frac{k_{12} \alpha_{2}\left(\mu_{0}-\mu\right)}{\lambda_{1} k_{1}-k_{11}\left(\mu_{0}-\mu\right)}>0 .
$$

\subsection{Local stability of equilibria}

In this section, the local stability analysis of equilibria of the model (5.1) is performed. The Jacobian of $(5.1)$ is $\widetilde{J}=\left[\widetilde{J}_{i j}\right], i, j=1,2,3,4$, with nonvanishing explicit entries given by

$$
\begin{aligned}
& \widetilde{J}_{11}=-\left(\alpha_{0}+\frac{\beta_{1} \beta_{12} A}{\left(\beta_{12}+\beta_{11} N\right)^{2}}\right), \widetilde{J}_{12}=-\frac{\beta_{1} N}{\beta_{12}+\beta_{11} N}, \widetilde{J}_{13}=\frac{\pi k_{1} k_{12} B}{\left(k_{12}+k_{11} D\right)^{2}}, \\
& \widetilde{J}_{14}=\frac{\pi k_{1} D}{k_{12}+k_{11} D}, \widetilde{J}_{21}=\frac{\theta_{1} \beta_{1} \beta_{12} A}{\left(\beta_{12}+\beta_{11} N\right)^{2}}, \widetilde{J}_{22}=\frac{\theta_{1} \beta_{1} N}{\beta_{12}+\beta_{11} N}-\alpha_{1}-2 \beta_{10} A, \\
& \widetilde{J}_{32}=\pi_{1}\left(\alpha_{1}+2 \beta_{10} A\right), \widetilde{J}_{33}=-\left(\alpha_{2}+\frac{k_{1} k_{12} B}{\left(k_{12}+k_{11} D\right)^{2}}\right), \widetilde{J}_{34}=-\frac{k_{1} D}{k_{12}+k_{11} D}, \\
& \widetilde{J}_{43}=\frac{\lambda_{1} k_{1} k_{12} B}{\left(k_{12}+k_{11} D\right)^{2}}, \widetilde{J}_{44}=\frac{\lambda_{1} k_{1} D}{k_{12}+k_{11} D}-\left(\mu_{0}-\mu\right) .
\end{aligned}
$$

1. The Jacobian $\widetilde{J}$ evaluated at the equilibrium $\widetilde{E}_{0}$ leads to the eigenvalues $-\alpha_{0},-\alpha_{1},-\alpha_{2}$ and $-\left(\mu_{0}-\mu\right)$. Since all the eigenvalues are negative, the equilibrium $\widetilde{E}_{0}$ is stable.

2. The Jacobian $\widetilde{J}$ evaluated at $\widetilde{E}^{*}$ leads to the matrix $\widetilde{J}_{\widetilde{E}^{*}}=\left[\widetilde{a}_{i j}\right], i, j=1,2,3$, 4. Some entries of matrix $\widetilde{J}_{\widetilde{E}^{*}}$ simplify as follows: $\left(\widetilde{J}_{\widetilde{E}^{*}}\right)_{22}=\widetilde{a}_{22}=-\beta_{10} \widetilde{A}^{*},\left(\widetilde{J}_{\widetilde{E}^{*}}\right)_{44}=\widetilde{a}_{44}=0$. 
and $A^{*}$ is positive root of the equation

$$
\begin{gathered}
G(A)=\left[q_{1}+\left(\pi \pi_{1}-\frac{1}{\theta_{1}}\right) \alpha_{1} A+\left(\pi \pi_{1}-\frac{1}{\theta_{1}}\right) \beta_{10} A^{2}\right]\left[\left(\theta_{1} \beta_{1}-\beta_{11} \alpha_{1}\right)-\beta_{11} \beta_{10} A\right] \\
-\beta_{12} \alpha_{0}\left(\alpha_{1}+\beta_{10} A\right)=0
\end{gathered}
$$


where $q_{1}=q-\frac{k_{12} \alpha_{2}\left(\mu_{0}-\mu\right)}{\lambda_{1} k_{1}-k_{11}\left(\mu_{0}-\mu\right)}$.

Positivity of $N^{*}$ and $B^{*}$ imply respectively the feasibility conditions

$$
\begin{aligned}
\left(\theta_{1} \beta_{1}-\beta_{11} \alpha_{1}\right) q_{1}-\beta_{12} \alpha_{1} \alpha_{0} & >0, \\
\pi_{1}\left(\alpha_{1} A^{*}+\beta_{10} A^{* 2}\right)-\frac{k_{12} \alpha_{2}\left(\mu_{0}-\mu\right)}{\lambda_{1} k_{1}-k_{11}\left(\mu_{0}-\mu\right)} & >0 .
\end{aligned}
$$

In addition, requiring

$$
q\left[\lambda_{1} k_{1}-k_{11}\left(\mu_{0}-\mu\right)\right]-k_{12} \pi \alpha_{2}\left(\mu_{0}-\mu\right)>0,
$$

ensures that $D^{*}>0$ and together with (6.4) implies that $G(0)>0$. Further, letting

$$
\bar{A}=\frac{\theta_{1} \beta_{1}-\beta_{11} \alpha_{1}}{\beta_{11} \beta_{10}}
$$

we find $G(\bar{A})<0$ and $G^{\prime}(A)<0$ for $A \in(0, \bar{A})$.

Thus there exists a unique positive $\operatorname{root} A=A^{*}$ of equation (6.3) in the interval $(0, \bar{A})$.

\subsection{Local stability of equilibria}

In this section, the local stability analysis of equilibria of the model (2.1) is performed. The Jacobian of $(2.1)$ is $J=\left[J_{i j}\right], i, j=1,2,3,4$, with nonvanishing explicit entries given by

$$
\begin{aligned}
& J_{11}=-\left(\alpha_{0}+\frac{\beta_{1} \beta_{12} A}{\left(\beta_{12}+\beta_{11} N\right)^{2}}\right), J_{12}=-\frac{\beta_{1} N}{\beta_{12}+\beta_{11} N}, J_{13}=\frac{\pi k_{1} k_{12} B}{\left(k_{12}+k_{11} D\right)^{2}}, \\
& J_{14}=\frac{\pi k_{1} D}{k_{12}+k_{11} D}, J_{21}=\frac{\theta_{1} \beta_{1} \beta_{12} A}{\left(\beta_{12}+\beta_{11} N\right)^{2}}, J_{22}=\frac{\theta_{1} \beta_{1} N}{\beta_{12}+\beta_{11} N}-\alpha_{1}-2 \beta_{10} A, \\
& J_{32}=\pi_{1}\left(\alpha_{1}+2 \beta_{10} A\right), J_{33}=-\left(\alpha_{2}+\frac{k_{1} k_{12} B}{\left(k_{12}+k_{11} D\right)^{2}}\right), J_{34}=-\frac{k_{1} D}{k_{12}+k_{11} D}, \\
& J_{43}=\frac{\lambda_{1} k_{1} k_{12} B}{\left(k_{12}+k_{11} D\right)^{2}}, \quad J_{44}=\frac{\lambda_{1} k_{1} D}{k_{12}+k_{11} D}-\left(\mu_{0}-\mu\right) .
\end{aligned}
$$

Theorem 6.1. 1. The equilibrium $E_{0}$ is unstable whenever the equilibrium $E_{1}$ is feasible.

2. The equilibrium $E_{1}$ is unstable whenever the equilibrium $E^{*}$ is feasible.

3. The equilibrium $E^{*}$, if feasible, is locally asymptotically stable provided the following conditions are satisfied, where the relevant quantities are defined in the proof:

$$
A_{4}>0, A_{1} A_{2}-A_{3}>0, A_{3}\left(A_{1} A_{2}-A_{3}\right)-A_{1}^{2} A_{4}>0 .
$$

Proof. 1. The Jacobian $J$ evaluated at the equilibrium $E_{0}$ leads to the following eigenvalues:

$$
-\alpha_{0}, \frac{\theta_{1} \beta_{1} q}{\beta_{12} \alpha_{0}+\beta_{11} q}-\alpha_{1},-\alpha_{2},-\left(\mu_{0}-\mu\right) .
$$

The second one is negative (or positive) provided condition (6.2) does not hold (or hold). Thus, the equilibrium $E_{0}$ is stable (or unstable) whenever the equilibrium $E_{1}$ is not feasible (or feasible).

2. The Jacobian $J$ evaluated at the equilibrium $E_{1}$ immediately gives two eigenvalues

$$
-\alpha_{2}, \frac{\lambda_{1} k_{1}\left(\pi_{1} \alpha_{1} A_{1}+\pi_{2} \beta_{10} A_{1}^{2}\right)}{k_{12} \alpha_{2}+k_{11} \pi_{1}\left(\alpha_{1} A_{1}+\beta_{10} A_{1}^{2}\right)}-\left(\mu_{0}-\mu\right)
$$


while the remaining two are roots of the following equation with all positive coefficients:

$$
\lambda^{2}+\left(\beta_{10} A_{1}+\alpha_{0}+\frac{\beta_{1} \beta_{12} A_{1}}{\left(\beta_{12}+\beta_{11} N_{1}\right)^{2}}\right) \lambda+\beta_{10} A_{1}+\frac{\theta_{1} \beta_{1} \beta_{12} A_{1}}{\left(\beta_{12}+\beta_{11} N_{1}\right)^{2}} \beta_{1} N_{1} \beta_{12}+\beta_{11} N_{1}=0 .
$$

Clearly, roots of equation (6.8) are either negative or with negative real parts. Thus, the matrix $J_{E_{1}}$ has three eigenvalues which are either negative or with negative real parts and one eigenvalue is negative (or positive) provided condition (6.5) is not satisfied (or satisfied). Therefore, the equilibrium $E_{1}$ is stable (or unstable) whenever the equilibrium $E^{*}$ is not feasible (or feasible).

3. The Jacobian $J$ evaluated at $E^{*}$ leads to the matrix $J_{E^{*}}=\left[a_{i j}\right], i, j=1,2,3$, 4 . Some entries of matrix $J_{E^{*}}$ simplify as follows: $\left(J_{E^{*}}\right)_{22}=a_{22}=-\beta_{10} A^{*},\left(J_{E^{*}}\right)_{44}=a_{44}=0$.

The associated characteristic equation is given by $\lambda^{4}+A_{1} \lambda^{3}+A_{2} \lambda^{2}+A_{3} \lambda+A_{4}=0$, where

$$
\begin{aligned}
& A_{1}=-\left(a_{11}+a_{22}+a_{33}\right), A_{2}=a_{11}\left(a_{22}+a_{33}\right)-a_{22} a_{33}-a_{34} a_{43}-a_{12} a_{21}, \\
& A_{3}=a_{11}\left(a_{22} a_{33}+a_{34} a_{43}\right)+a_{22} a_{34} a_{43}+a_{21}\left(a_{12} a_{33}-a_{13} a_{32}\right), \\
& A_{4}=a_{21}\left(a_{12} a_{34} a_{43}-a_{14} a_{34} a_{43}\right)-a_{11} a_{22} a_{34} a_{43} .
\end{aligned}
$$

Clearly, $A_{1}$ is positive. Using the Routh-Hurwitz criterion, roots of the characteristic equation are either negative or have negative real parts iff conditions (6.7) are satisfied.

\section{Transcritical bifurcation}

Considering $E_{0}$ and $E_{1}$ taking $\beta_{1}$ as a bifurcation parameter, then at $\beta_{1}=\beta_{1}^{*}$, an exchange of feasibility and stability properties between these two equilibria occurs. This is a clear indication of the presence of a transcritical bifurcation at the critical threshold $\beta_{1}^{*}$. We now rigorously prove that indeed this is the case.

Observe that the eigenvalues of the Jacobian matrix

$$
J\left(E_{0}, \beta_{1}^{*}\right)=\left(\begin{array}{cccc}
-\alpha_{0} & -\frac{\beta_{1} q}{\beta_{12} \alpha_{0}+\beta_{11} q} & 0 & 0 \\
0 & 0 & 0 & 0 \\
0 & \pi_{1} \alpha_{1} & -\alpha_{2} & 0 \\
0 & 0 & 0 & -\left(\mu_{0}-\mu\right)
\end{array}\right),
$$

are given by

$$
\eta_{1}=-\alpha_{0}, \eta_{2}=0, \eta_{3}=-\alpha_{2} \text { and } \eta_{4}=-\left(\mu_{0}-\mu\right) .
$$

Thus, $\eta_{2}=0$ is a simple zero eigenvalue and the other ones are real and negative. Hence, at $\beta_{1}=\beta_{1}^{*}$ the equilibrium $E_{0}$ is non-hyperbolic and the assumption (A1) of Theorem 4.1 in (56) is verified.

Now, denote by $\mathbf{w}=\left(w_{1}, w_{2}, w_{3}, w_{4}\right)^{T}$ a right eigenvector associated with the zero eigenvalue $\eta_{2}=0$, explicitly given by

$$
w_{1}=-\frac{\beta_{1} q}{\alpha_{0}\left(\beta_{12} \alpha_{0}+\beta_{11} q\right)}, w_{2}=1, w_{3}=\frac{\pi_{1} \alpha_{1}}{\alpha_{2}} \text { and } w_{4}=0
$$

Furthermore, the left eigenvector $\mathbf{v}=\left(v_{1}, v_{2}, v_{3}, v_{4}\right)$ is $\mathbf{v}=(0,1,0,0)$, so that $\mathbf{w} \cdot \mathbf{v}=1$.

Now, the coefficients $a$ and $b$ defined in Theorem 4.1 of (56)

$$
a=\sum_{k, i, j=1}^{4} v_{k} w_{i} w_{j} \frac{\partial^{2} f_{k}}{\partial x_{i} \partial x_{j}}\left(E_{0}, \beta_{1}^{*}\right), b=\sum_{k, i=1}^{4} v_{k} w_{i} \frac{\partial^{2} f_{k}}{\partial x_{i} \partial \beta_{1}}\left(E_{0}, \beta_{1}^{*}\right),
$$


may be explicitly computed. Taking into account system (2.1), it follows that

$$
a=\frac{\beta_{12} \alpha_{0} \alpha_{1}}{\theta_{1} q^{2}\left(\beta_{12} \alpha_{0}+\beta_{11} q\right)}-2 \beta_{10}, \quad b=\frac{\theta_{1} q}{\beta_{12} \alpha_{0}+\beta_{11} q}>0 .
$$

Now $a>0$ if and only if

$$
\frac{\beta_{12} \alpha_{0} \alpha_{1}}{\theta_{1} q^{2}\left(\beta_{12} \alpha_{0}+\beta_{11} q\right)}>2 \beta_{10}
$$

In view of previous considerations, we have the following theorem.

Theorem 7.1. Consider system (2.1) and let $a$ and $b$ as given by (7.1), where $b>0$. The local dynamics of system (2.1) around the equilibrium $E_{0}$ are totally determined by the sign of a.

(i) If $a<0$, when $\beta_{1}<\beta_{1}^{*}$ with $\beta_{1} \approx \beta_{1}^{*}, E_{0}$ is locally asymptotically stable, and there exists a negative unstable equilibrium $E_{1}$; when $\beta_{1}>\beta_{1}^{*}$ with $\beta_{1} \approx \beta_{1}^{*}, E_{0}$ is unstable, and there exists a positive locally asymptotically stable equilibrium $E_{1}$.

(ii) If $a>0$, when $\beta_{1}<\beta_{1}^{*}$ with $\beta_{1} \approx \beta_{1}^{*}, E_{0}$ is locally asymptotically stable, and there exists a positive unstable equilibrium $E_{1}$; when $\beta_{1}>\beta_{1}^{*}$ with $\beta_{1} \approx \beta_{1}^{*}, E_{0}$ is unstable, and there exists a negative locally asymptotically stable equilibrium $E_{1}$.

Proof. It follows from (56) Theorem 4.1 pp. 373, and Remark 1 pp. 375.

Corollary 7.1. Consider the model (2.1) and let $a$ and $b$ as given by (7.1) where $b>0$. At $\beta_{1}=\beta_{1}^{*}$, the system (2.1) undergoes a transcritical bifurcation. If $a<0$ the bifurcation at $\beta_{1}=\beta_{1}^{*}$ is supercritical (or forward). On the other hand, if $a>0$, the bifurcation at $\beta_{1}=\beta_{1}^{*}$ is subcritical (or backward) bifurcation.

Proof. It is a straightforward application of Theorem 7.1.

Remark 1. Taking $\mu_{0}$ as a bifurcation parameter, we can prove the existence of a transcritical bifurcation between equilibria $E_{1}$ and $E^{*}$ of the system (2.1) using the same approach as above.

In Table 2, we listed the equilibria of the systems (2.1), (3.1), (4.1) and (5.1), and the conditions for their feasibility and stability.

\begin{tabular}{|c|c|c|c|}
\hline Models & Equilibria & Feasibility conditions & Conditions for LAS \\
\hline$(3.1)$ & $\begin{array}{l}\bar{E}_{0}=\left(q \alpha_{0}^{-1}, 0\right) \\
\bar{E}^{*}=\left(\bar{N}^{*}, \bar{A}^{*}\right)\end{array}$ & $\begin{array}{c}\text { Always feasible } \\
q\left(\theta_{1} \beta_{1}-\beta_{11} \alpha_{1}\right)-\beta_{12} \alpha_{1} \alpha_{0}>0\end{array}$ & $\begin{aligned} & q\left(\theta_{1} \beta_{1}-\right.\left.\beta_{11} \alpha_{1}\right)-\beta_{12} \alpha_{1} \alpha_{0}<0 \\
& \text { Always stable }\end{aligned}$ \\
\hline$(4.1)$ & $\begin{array}{c}\widehat{E}_{0}=\left(q \alpha_{0}^{-1}, 0,0\right) \\
\widehat{E}^{*}=\left(\widehat{N}^{*}, \widehat{A}^{*}, \widehat{D}^{*}\right)\end{array}$ & $\begin{array}{c}\text { Always feasible } \\
q\left(\theta_{1} \beta_{1}-\beta_{11} \alpha_{1}\right)-\beta_{12} \alpha_{1} \alpha_{0}>0\end{array}$ & $\begin{array}{c}q\left(\theta_{1} \beta_{1}-\beta_{11} \alpha_{1}\right)-\beta_{12} \alpha_{1} \alpha_{0}<0 \\
\text { Always stable }\end{array}$ \\
\hline$(5.1)$ & $\begin{array}{c}\widetilde{E}_{0}=(0,0,0,0) \\
\widetilde{E}^{*}=\left(\widetilde{N}^{*}, \widetilde{A}^{*}, \widetilde{D}^{*}, \widetilde{B}^{*}\right)\end{array}$ & $\begin{array}{c}\text { Always feasible } \\
\pi_{1} \widetilde{A}^{*}\left(\alpha_{1}+\beta_{10} \widetilde{A}^{*}\right)-\frac{k_{12} \alpha_{2}\left(\mu_{0}-\mu\right)}{\lambda_{1} k_{1}-k_{11}\left(\mu_{0}-\mu\right)}>0 \\
\left(\theta_{1} \beta_{1}-\beta_{11} \alpha_{1}\right)-\beta_{11} \beta_{10} \widetilde{A}^{*}>0 \\
\theta_{1} \beta_{1}-2 \beta_{11} \alpha_{1}>0\end{array}$ & $\begin{array}{c}\text { Always stable } \\
\widetilde{A}_{4}>0, \quad \widetilde{A}_{1} \widetilde{A}_{2}-\widetilde{A}_{3}>0 \\
\widetilde{A}_{3}\left(\widetilde{A}_{1} \widetilde{A}_{2}-\widetilde{A}_{3}\right)-\widetilde{A}_{1}^{2} \widetilde{A}_{4}>0\end{array}$ \\
\hline$(2.1)$ & $\begin{array}{c}E_{0}=\left(q \alpha^{-1}, 0,0,0\right) \\
E_{1}=\left(N_{1}, A_{1}, D_{1}, 0\right) \\
E^{*}=\left(N^{*}, A^{*}, D^{*}, B^{*}\right)\end{array}$ & $\begin{array}{c}\text { Always feasible } \\
q\left(\theta_{1} \beta_{1}-\beta_{11} \alpha_{1}\right)-\beta_{12} \alpha_{1} \alpha_{0}>0 \\
\pi_{1}\left(\alpha_{1} A^{*}+\beta_{10} A^{* 2}\right)-\frac{k_{12} \alpha_{2}\left(\mu_{0}-\mu\right)}{\lambda_{1} k_{1}-k_{11}\left(\mu_{0}-\mu\right)}>0 \\
\left(\theta_{1} \beta_{1}-\beta_{11} \alpha_{1}\right) q_{1}-\beta_{12} \alpha_{1} \alpha_{0}>0 \\
q\left[\lambda_{1} k_{1}-k_{11}\left(\mu_{0}-\mu\right)\right]-k_{12} \pi \alpha_{2}\left(\mu_{0}-\mu\right)>0\end{array}$ & $\begin{array}{c}q\left(\theta_{1} \beta_{1}-\beta_{11} \alpha_{1}\right)-\beta_{12} \alpha_{1} \alpha_{0}<0 \\
\pi_{1}\left(\alpha_{1} A_{1}+\beta_{10} A_{1}^{2}\right)<\frac{k_{12} \alpha_{2}\left(\mu_{0}-\mu\right)}{\lambda_{1} k_{1}-k_{11}\left(\mu_{0}-\mu\right)} \\
A_{4}>0, A_{1} A_{2}-A_{3}>0 \\
A_{3}\left(A_{1} A_{2}-A_{3}\right)-A_{1}^{2} A_{4}>0\end{array}$ \\
\hline
\end{tabular}

Table 2: Equilibria and conditions for their feasibility and stability 
Remark 2. Since the inflow rate of nutrients in the aquatic system plays an important role in determining the dynamics of the system, Table 2 allows to assess how system dynamics is affected by changes in the input rate of nutrients. Note that the equilibrium $\widetilde{E_{0}}$ is always feasible and stable while the equilibrium $\widetilde{E}^{*}$ is feasible if conditions (5.3) and (5.4) are satisfied, and is stable provided conditions in (5.5) hold. That is, if conditions (5.3), (5.4) and (5.5) are satisfied, the equilibria $\widetilde{E}_{0}$ and $\widetilde{E}^{*}$ are feasible and stable simultaneously. This ensures bistability. The bistability behavior of system (5.1) is shown in Fig. 2. Thus, by completely stopping the input rate of nutrients in the lake, we may achieve the algae-free system or algae-persistent system depending on the current value of the algal density. Recall that when $q \neq 0$, the algae-free equilibrium is stable only when the algae-persistent equilibrium is not feasible. Overall, in order to preserve the aquatic system, the inflow rate of the nutrients coming from various sources must be controlled. Moreover, for the systems without bacteria, (i.e. systems (3.1) and (4.1)), the coexistence equilibrium is always locally asymptotically stable and globally asymptotically stable under certain conditions.

\section{Effect of time delay}

In this section, we modify our model (2.1) by incorporating a discrete time delay which represents the time lag involved in the conversion of detritus into nutrients due to bacterial decomposition and the corresponding growth in the bacterial population. For instance, we consider the same lag $(\tau)$ in these two processes. With this modification, we have the following system of delay differential equations:

$$
\begin{aligned}
\frac{d N(t)}{d t} & =q-\alpha_{0} N(t)-\frac{\beta_{1} N(t) A(t)}{\beta_{12}+\beta_{11} N(t)}+\frac{\pi k_{1} D(t-\tau) B(t)}{k_{12}+k_{11} D(t-\tau)}, \\
\frac{d A(t)}{d t} & =\frac{\theta_{1} \beta_{1} N(t) A(t)}{\beta_{12}+\beta_{11} N(t)}-\alpha_{1} A(t)-\beta_{10} A^{2}(t), \\
\frac{d D(t)}{d t} & =\pi_{1}\left(\alpha_{1} A(t)+\beta_{10} A^{2}(t)\right)-\alpha_{2} D(t)-\frac{k_{1} D(t) B(t)}{k_{12}+k_{11} D(t)} \\
\frac{d B(t)}{d t} & =\mu B(t)-\mu_{0} B(t)+\frac{\lambda_{1} k_{1} D(t-\tau) B(t)}{k_{12}+k_{11} D(t-\tau)} .
\end{aligned}
$$

The initial conditions for the system (8.1) take the form

$$
N(\phi)=\psi_{1}(\phi), \quad P(\phi)=\psi_{2}(\phi), \quad A(\phi)=\psi_{3}(\phi), \quad D(\phi)=\psi_{4}(\phi), \quad-\tau \leq \phi \leq 0,
$$

where $\psi=\left(\psi_{1}, \psi_{2}, \psi_{3}, \psi_{4}\right)^{T} \in \mathcal{C}_{+}$such that $\psi_{i}(\phi) \geq 0, i=1,2,3,4 \forall \phi \in[-\tau, 0]$ and $\mathcal{C}_{+}$denotes the Banach space $\mathcal{C}_{+}\left([-\tau, 0], \mathbf{R}_{+0}^{4}\right)$ of continuous functions mapping the interval $[-\tau, 0]$ into $\mathbf{R}_{+0}^{4}$. The norm of an element $\psi$ in $\mathcal{C}_{+}$is denoted by $\|\psi\|=\sup _{-\tau \leq \phi \leq 0}\left\{\left|\psi_{1}(\phi)\right|,\left|\psi_{2}(\phi)\right|,\left|\psi_{3}(\phi)\right|,\left|\psi_{4}(\phi)\right|\right\}$. For biological feasibility, we further assume that $\psi_{i}(0) \geq 0$ for $i=1,2,3,4$.

In the following, we carry out the local stability analysis for the interior equilibrium $E^{*}$ and show that the system (8.1) undergoes a Hopf-bifurcation around this equilibrium.

\section{Hopf-bifurcation analysis}

To study the stability behavior of the equilibrium $E^{*}$ in the presence of time delay, we linearize the system (8.1) about the equilibrium $E^{*}$ and get

$$
\frac{d Y}{d t}=L Y(t)+M Y(t-\tau)
$$


where

$$
L=\left(\begin{array}{cccc}
V_{11} & V_{12} & 0 & V_{14} \\
V_{21} & V_{22} & 0 & 0 \\
0 & V_{32} & V_{33} & V_{34} \\
0 & 0 & 0 & 0
\end{array}\right), M=\left(\begin{array}{cccc}
0 & 0 & M_{13} & 0 \\
0 & 0 & 0 & 0 \\
0 & 0 & 0 & 0 \\
0 & 0 & M_{43} & 0
\end{array}\right) \text { and } Y(\cdot)=\left(\begin{array}{c}
n(\cdot) \\
a(\cdot) \\
d(\cdot) \\
b(\cdot)
\end{array}\right)
$$

with

$$
\begin{aligned}
& V_{11}=-\left(\alpha_{0}+\frac{\beta_{1} \beta_{12} A^{*}}{\left(\beta_{12}+\beta_{11} N^{*}\right)^{2}}\right), V_{12}=-\frac{\beta_{1} N^{*}}{\beta_{12}+\beta_{11} N^{*}}, V_{14}=\frac{\pi k_{1} D^{*}}{k_{12}+k_{11} D^{*}}, \\
& V_{21}=\frac{\theta_{1} \beta_{1} \beta_{12} A^{*}}{\left(\beta_{12}+\beta_{11} N^{*}\right)^{2}}, V_{22}=-\beta_{10} A^{*}, V_{32}=\pi_{1}\left(\alpha_{1}+2 \beta_{10} A^{*}\right), \\
& V_{33}=-\left(\alpha_{2}+\frac{k_{1} k_{12} B^{*}}{\left(k_{12}+k_{11} D^{*}\right)^{2}}\right), V_{34}=-\frac{k_{1} D^{*}}{k_{12}+k_{11} D^{*}}, M_{13}=\frac{\pi k_{1} k_{12} B^{*}}{\left(k_{12}+k_{11} D^{*}\right)^{2}}, M_{43}=\frac{\lambda_{1} k_{1} k_{12} B^{*}}{\left(k_{12}+k_{11} D^{*}\right)^{2}} .
\end{aligned}
$$

Here, $n, a, d$ and $b$ are small perturbations around the equilibrium $E^{*}$. The characteristic equation for the linearized system (9.1) is given by

$$
\lambda^{4}+A_{3} \lambda^{3}+A_{2} \lambda^{2}+A_{1} \lambda+\left(B_{2} \lambda^{2}+B_{1} \lambda+B_{0}\right) e^{-\lambda \tau}=0,
$$

where

$$
\begin{aligned}
& A_{3}=-\left(V_{11}+V_{22}+V_{33}\right), A_{2}=V_{11} V_{22}+V_{22} V_{33}+V_{11} V_{33}-V_{12} V_{21}, \\
& A_{1}=V_{12} V_{21} V_{33}-V_{11} V_{22} V_{33}, B_{2}=-V_{34} M_{43}, \\
& B_{1}=V_{22} V_{34} M_{43}+V_{11} V_{34} M_{43}-V_{21} V_{33} M_{13}, B_{0}=V_{12} V_{21} V_{34} M_{43}+V_{14} V_{21} V_{32} M_{43}-V_{11} V_{22} V_{34} M_{43} .
\end{aligned}
$$

Equation (9.2) is transcendental in $\lambda$, so that it has infinitely many complex roots. To understand the local stability behavior of the equilibrium $E^{*}$, we need to assess the signs of real parts of the roots of equation (9.2). This is a complicated task in the presence of the time delay. Therefore, equation (9.2) is first analyzed in the absence of time delay and then conditions for local asymptotic stability behavior of the equilibrium $E^{*}$ are deduced when time delay is present.

For $\tau=0$, Theorem 6.1 provides the conditions under which all the roots of equation (9.2) are either negative or with negative real parts. For $\tau>0$ by Rouche's Theorem and continuity in $\tau$, the sign of roots of equation (9.2) will change across the imaginary axis, i.e., if equation (9.2) has purely imaginary roots. Hence, putting $\lambda=i \omega(\omega>0)$ in equation (9.2) and separating real and imaginary parts, we get

$$
\begin{aligned}
w^{4}-A_{2} \omega^{2} & =-\left(B_{0}-B_{2} \omega^{2}\right) \cos (\omega \tau)-B_{1} \omega \sin (\omega \tau), \\
A_{3} \omega^{3}-A_{1} \omega & =-\left(B_{0}-B_{2} \omega^{2}\right) \sin (\omega \tau)+B_{1} \omega \cos (\omega \tau) .
\end{aligned}
$$

Squaring and adding equations (9.3) and (9.4), we obtain

$$
\left(w^{4}-A_{2} \omega^{2}\right)^{2}+\left(A_{3} \omega^{3}-A_{1} \omega\right)^{2}=\left(B_{0}-B_{2} \omega^{2}\right)^{2}+B_{1}^{2} \omega^{2} .
$$

Simplifying equation (9.5) and substituting $\omega^{2}=\psi$, we get the following equation in $\psi$ :

$$
\Psi(\psi)=\psi^{4}+C_{3} \psi^{3}+C_{2} \psi^{2}+C_{1} \psi+C_{0}=0,
$$

where

$$
C_{3}=-2 A_{2}+A_{3}^{2}, C_{2}=A_{2}^{2}-2 A_{1} A_{3}-B_{2}^{2}, C_{1}=A_{1}^{2}+2 B_{2} B_{0}-B_{1}^{2}, C_{0}=-B_{0}^{2} .
$$


The existence of positive roots of equation (9.6) is addressed in the following lemma, whose proof follows by Descartes' rule, (57).

Lemma 2. The polynomial equation (9.6) has

(1) at least one positive root if

$$
\begin{aligned}
& \text { (a) } C_{3}>0, C_{2}<0, C_{1}>0, C_{0}<0 . \\
& \text { (b) } C_{3}<0, C_{2}<0, C_{1}>0, C_{0}<0 . \\
& \text { (c) } C_{3}<0, C_{2}>0, C_{1}>0, C_{0}<0 . \\
& \text { (d) } C_{3}<0, C_{2}>0, C_{1}<0, C_{0}<0 .
\end{aligned}
$$

(2) exactly one positive root if

$$
\begin{aligned}
& \text { (a) } C_{3}<0, C_{2}<0, C_{1}<0, C_{0}<0 . \\
& \text { (b) } C_{3}>0, C_{2}<0, C_{1}<0, C_{0}<0 . \\
& \text { (c) } C_{3}>0, C_{2}>0, C_{1}<0, C_{0}<0 . \\
& \text { (d) } C_{3}>0, C_{2}>0, C_{1}>0, C_{0}<0 .
\end{aligned}
$$

For other choices of the coefficients of equation (9.6), the positive root cannot be guaranteed.

Now, we can characterize the system behavior:

Theorem 9.1. Assume that the equilibrium $E^{*}$ is locally asymptotically stable for $\tau=0$ and that one of the conditions (1) or (2) given in Lemma 2 holds. Let $\psi_{0}=\omega_{0}^{2}$ be a positive root of (9.6). Then, there exists $\tau=\tau_{0}$ such that the equilibrium $E^{*}$ is asymptotically stable when $0 \leq \tau<\tau_{0}$ and unstable for $\tau>\tau_{0}$, where

$$
\tau_{k}=\frac{1}{\omega_{0}} \tan ^{-1}\left[\frac{B_{1} \omega_{0}\left(\omega_{0}^{4}-A_{2} \omega_{0}^{2}\right)+\left(B_{0}-B_{2} \omega_{0}^{2}\right)\left(A_{3} \omega_{0}^{3}-A_{1} \omega_{0}\right)}{\left(B_{0}-B_{2} \omega_{0}^{2}\right)\left(\omega_{0}^{4}-A_{2} \omega_{0}^{2}\right)-B_{1} \omega_{0}\left(A_{3} \omega_{0}^{3}-A_{1} \omega_{0}\right)}\right]+\frac{k \pi}{\omega_{0}},
$$

for $k=0,1,2,3 \cdots$. Furthermore, the system will undergo a Hopf-bifurcation at $E^{*}$ when $\tau=\tau_{0}$ provided $\Psi^{\prime}\left(\omega_{0}^{2}\right)>0$.

Proof. Since $\psi=\omega_{0}^{2}$ is a solution of the equation (9.6), the characteristic equation (9.2) has pair of purely imaginary roots $\pm i \omega_{0}^{2}$. It follows from equations (9.3) and (9.4) that $\tau_{k}$ is a function of $\omega_{0}^{2}$ for $k=0,1,2,3 \cdots$. Therefore, the system will be locally asymptotically stable at $E^{*}$ for $\tau=0$, if the conditions (6.7) hold. In that case by Butler's lemma, the equilibrium $E^{*}$ will remain stable for $\tau<\tau_{0}$, such that $\tau_{0}=\min _{k \geq 0} \tau_{k}$ and unstable for $\tau \geq \tau_{0}$, provided that the transversality condition holds. The transversality condition is given as

$$
\operatorname{sgn}\left[\frac{d(\operatorname{Re}(\lambda))}{d \tau}\right]_{\tau=\tau_{0}}^{-1}=\left[\frac{\Psi^{\prime}\left(\omega_{0}^{2}\right)}{B_{1}^{2} \omega_{0}^{2}+\left(B_{0}-B_{2} \omega_{0}^{2}\right)^{2}}\right] .
$$

Note that $\Psi^{\prime}\left(\omega_{0}^{2}\right) \neq 0$ if Lemma 2 holds. Hence, the transversality condition is satisfied and a Hopf bifurcation occurs at $\tau=\tau_{0}$ i.e., a family of periodic solutions emanate from the equilibrium $E^{*}$ as the delay parameter, $\tau$, passes through its critical value, $\tau_{0},(31)$.

\section{Numerical simulations}

Here, we report the simulations performed to investigate the system behavior using the Matlab variable step Runge-Kutta solver ode45. In spite of importance of clean water on the Earth, quantitative 
data on water pollution are scanty and, therefore, we are unable to check the validity of the model and its outcome with the field data. However, to visualize different analytical results and to have some insights from it, we have numerically simulated the system (2.1). The (hypothetical) parameter values are chosen within ranges defined in the existing literature $(6 ; 9 ; 40)$.

First of all, we choose some important parameters of the model (2.1) and see their effects on the equilibrium values of nutrients, algae, detritus and bacteria in the lake. For our purpose, we select $q, \beta_{1}$, $\beta_{10}, k_{1}, \mu$ and $\mu_{0}$ and analyze the behavior of model's variables by varying two parameters at a time viz. $\left(q, k_{1}\right),\left(\mu_{0}, \beta_{10}\right)$ and $\left(\mu, \beta_{1}\right)$ integrating up to time $t=100$ days, when the system is stabilized. In each figure, the surface represents the value of the population at a (dynamic) equilibrium, i.e. steady state or persistent oscillation. When two surfaces are shown, they indicate the maximum and minimum values that these variables attain in the limit cycle. When they collide, it means that a stable equilibrium is attained, while when they differ, the solution oscillates.

Fig. 3 shows the system behavior as functions of $q$ and $k_{1}$. On increasing the input rate of nutrients in the lake, the equilibrium values of all the populations increase. In this case, sustained oscillations in time are found $(34 ; 58)$. For low values of $k_{1}$, the bacteria vanish but this population increases with an increase in $k_{1}$. On the other hand, the detritus is high for low values of $k_{1}$, but as $k_{1}$ increases, bacteria decompose it at a high rate leading to a detritus-free environment.

In Fig. 4 , we vary $\mu_{0}$ and $\beta_{10}$. For low values of the bacteria mortality rate $\mu_{0}$, the bacterial population is at high level but disappears on increasing the values of $\mu_{0}$. Consequently the density of detritus increases at a high rate. On increasing the algae intraspecific competition rate $\beta_{10}$, the algal population decreases to a very low level while the nutrients increase as there are less algae that utilize them. Here too persistent oscillations are observed in all the panels.

Fig. 5 considers the parameters $\mu$ and $\beta_{1}$. Increase in $\beta_{1}$ causes a significant decrease in the concentration of nutrients but an opposite behavior for algae and detritus. For low values of $\beta_{1}$, these populations vanish altogether. Bacteria thrive only for larger values of $\mu$ and increase more with an increase in $\beta_{1}$. Again, we can observe persistent oscillations for all variables of the model.

We show the transcritical bifurcation between the equilibria $E_{0}$ and $E_{1}$ also numerically, varying the uptake rate of nutrients by algae, $\beta_{1}$, in Fig. 6(a). For low values of $\beta_{1}$ (i.e., no nutrients consumed by algae), the nutrients remains at a fixed level but their concentration suddenly drops as $\beta_{1}$ crosses its critical value. Also, there is a transcritical bifurcation between the equilibria $E_{1}$ and $E^{*}$, shown varying the natural death rate of bacteria, $\mu_{0}$, in Fig. 6(b). Actually, the coexistence equilibrium $E^{*}$ can stably achieved for the parameters values as given in Table 1, not shown.

To see the effect of the delay involved in the conversion of detritus into nutrients and the growth of bacterial populations, we set the system at a stable focus in the absence of time delay $(\tau=0)$. We gradually increase the value of time delay $\tau$ and observe the solution trajectories that still produce a stable focus at $\tau=10$ days, not shown. By increasing the time delay to $\tau=15.2$ days, the system (8.1) exhibits limit cycles, Fig. 7. Next, we increase the values of time delay to $\tau=20$ days and found that the system exhibits period doubling solutions, Fig. 8. The system shows chaotic behavior for further increase in time delay $(\tau=21.5$ days), Fig. 9. For better visualization, we draw the bifurcation diagram of the system (8.1) by varying the bifurcation parameter $\tau \in[10,22]$, Fig. 10. It is clear from the figure that the chaotic regime is reached via a stable focus for $\tau<14.5$, limit cycle behaviour for $14.5 \leq \tau \leq 15.9$, period doubling oscillations for $15.9 \leq \tau \leq 20.2$, and higher periodic and chaotic oscillations for $\tau \geq 20.2$. However, we could not provide bifurcation diagram for $\tau>22$, because the solutions trajectories of the system (2.1) blow up after $\tau>22$. Recall that systems in the absence of bacteria (systems (3.1) and (4.1)) are bounded while the systems in the presence of bacteria (systems (2.1) and (5.1)) are unbounded. Further, we draw the Poincaré map of the system (8.1) in the $A-D-B$ 
space fixing $N=0.8$, for $\tau=21.5$ days, Fig. 11. The scattered distribution of the sampling points implies the chaotic behavior of the system. We also draw the maximum Lyapunov exponent of the system (8.1) for $\tau=21.5$ days, Fig. 12. To draw the maximum Lyapunov exponent, we first simulate the delayed system (8.1). Then considering the times series solutions of each component, we compute the Lyapunov exponents by using the algorithm of $(59 ; 60)$. In the figure, positive values of the maximum Lyapunov exponent indicates the chaotic regime of the system. Therefore, we can conclude that the system (8.1) shows chaotic behavior for $\tau=21.5$ days.

\subsection{Sensitivity analysis}

We perform the global sensitivity analysis following the techniques of $(61 ; 62)$ to identify the most influential parameters that have significant impact on some important output variables of the system (2.1). We calculate partial rank correlation coefficients (PRCCs) between the parameters $q, \beta_{1}, k_{1}, \pi, \theta_{1}$, $\lambda_{1}, \mu$ and $\mu_{0}$ from system (2.1) with algae and bacteria in the lake as output. Nonlinear and monotone relationships were observed with the input parameters of the model (2.1), which is a prerequisite for computing PRCCs. Using the Latin Hypercube Sampling (LHS), we drawn 200 samples from the biologically feasible regions of the parameters of interest. The bar diagram of the PRCC values of the densities of algae and bacteria against these parameters is depicted in Fig. 13. PRCC values of these parameters with the responses suggest that the parameters $q, \beta_{1}, \theta_{1}, \mu$ and $\mu_{0}$ have significant correlations with the density of algae, Fig. 13(a). The parameter $\theta_{1}$ has maximum positive correlation with the density of algae. The bacteria mortality has negative correlation with the density of algae while other parameters have positive correlations. For the density of bacteria, the significant parameters are $\beta_{1}, \theta_{1}, \lambda_{1}, \mu$ and $\mu_{0}$, Fig. 13(b). The growth rate of bacteria due to detritus of other type than algal one, $\mu$, has maximum positive correlation with bacteria. The death rate of bacteria, $\mu_{0}$, has negative correlation with bacteria whereas other parameters have positive correlations.

\section{Discussion}

Marine microbes play important roles in aquatic systems, especially marine ones. They influence the climate, mediate primary production, participate in biogeochemical cycles, and maintain ecological balance (63). The microbes that make up harmful algal blooms are being studied closely by scientists (64). Because of high diversity and complexity, the interaction between algae and bacteria has become the concern of many researchers. Scientists have explored the relationships between algae and bacteria from different perspectives, including the physical, biological, environmental and chemical processes involved (64). Microbes, which exist as free-living forms as well as securely attached to algal cells, have now been demonstrated to modulate algal growth rates and transitions between life history stages, influence toxin production, and even induce the rapid lysis of algal cells (65). However, given the complex array of interactions that have evolved between them, some ecological functions and related mechanisms have not yet been fully elucidated.

In this paper, we have investigated the effect of bacteria on the dynamics of algal blooms in lakes. A nonlinear mathematical model has been proposed by taking nutrients, algae, detritus and bacteria as state variables. The model exhibits three non-negative equilibria; $E_{0}, E_{1}$ and $E^{*}$ that are related to each other via transcritical bifurcations. Equilibria $E_{0}$ and $E_{1}$ are linked on varying the parameter $\beta_{1}$, the latter arising when the bifurcation parameter $\beta_{1}$ crosses from below its critical value. On the other hand, the equilibria $E_{1}$ and $E^{*}$ are tied by the parameter $\mu_{0}$, the former arising when the bifurcation parameter $\mu_{0}$ crosses from below its critical value. For higher values of $\mu_{0}$, bacteria vanish and hence the density of algae in the lake increases up to a limit value. The partial rank correlation coefficient (PRCC) technique is performed to assess the sensitivity of the ecosystem with respect to the model parameters. 
The main parameters influencing the system behavior appear to be $q, \beta_{1}, k_{1}, \pi, \theta_{1}, \lambda_{1}$ and $\mu$. They present positive correlations with the densities of algae and bacterial population.

The effect of time delay on algal bloom into the lake has also been investigated. The time delay has been introduced to model more realistically the formation of nutrients from detritus and the subsequent growth of bacteria, that are not instantaneous processes. Previous studies show the occurrence of limit cycle oscillations through a Hopf-bifurcation by varying the delay parameter $(9 ; 40)$. However, to the best of our knowledge, in the context of algal bloom models no study is carried out for the delayed systems showing chaotic dynamics. In the present investigation, we found that as the delay parameter increases, the system (8.1) exhibits a transition from stable focus to limit cycle oscillations to period doubling oscillations to chaotic dynamics. The density of algae in the lake may increase drastically and in that case a massive death of fishes may occur as a consequence of low levels of dissolved oxygen. To avoid this unpleasant outcome, the detritus of the lake should be removed before the critical value of delay parameter is reached. Longer delay in this action may cause fluctuations in the density of algae for a long time and cause hypoxia in the lake. These findings are thus of critical importance for the practice of lake restoration.

The role of space in ecological interactions has been identified as an important factor (66). Spatial patterns are ubiquitous in nature and modify the stability properties of ecosystems at a range of spatial scales. Spatial variability of algae is very common in aquatic ecosystems. Several attempts have been made to explain such spatial variation using different types of mathematical models $(67 ; 68)$. To further extrapolate the results of the present study, extensions of the model presented here might be worth investigating, incorporating perhaps diffusion and advection, that play a crucial role in the spatial movement of algae in water bodies. A second issue concerns the parameter values used. Here they are taken as hypothetical, but tuning them using real data would a be very interesting step. At present however, for lack of the field results, is not possible. This investigation might be undertaken in future researches.

\section{Acknowledgements}

The authors thank the associate editor and anonymous reviewers for valuable comments, which contributed to the improvement in the presentation of the paper. Authors are grateful to Prof. Guido Badino, DBIOS, University of Turin, Italy for his valuable suggestions. Pankaj Kumar Tiwari is thankful to University Grants Commissions, New Delhi, India for providing financial support in form of Dr. D.S. Kothari post-doctoral fellowship (No.F.4-2/2006 (BSR)/MA/17-18/0021).

\section{References}

[1] D.M. Sigman, M.P. Hain, The biological productivity of the ocean. Nature Education Knowledge $3(10)$ (2012) 21.

[2] J. Lv, H. Wu, M. Chen, Effects of nitrogen and phosphorus on phytoplankton composition and biomass in 15 subtropical, urban shallow lakes in Wuhan, China. Limnologica, 41(1) (2011) 48-56.

[3] R. Ramanan et al., Algae-bacteria interactions: evolution, ecology and emerging applications. Biotechnology advances, 34(1) (2016) 14-29.

[4] The Effects: Dead Zones and Harmful Algal Blooms. https://www .epa.gov/nutrientpollution/effects-dead-zc 
[5] D. Anderson, HABs in a changing world: a perspective on harmful algal blooms, their impacts, and research and management in a dynamic era of climatic and environmental change, Harmful Algae 2012 (2014) 3-17.

[6] J.B. Shukla, A.K. Misra, P. Chandra, Modeling and analysis of the algal bloom in a lake caused by discharge of nutrients, Appl. Math. Comp. 196(2) (2008) 782-790.

[7] A.K. Misra, Modeling the depletion of dissolved oxygen in a lake due to submerged macrophytes, Nonlinear Anal. Model. Control 15(2) (2010) 185-198.

[8] A.K. Misra, P.K. Tiwari, E. Venturino, Modeling the impact of awareness on the mitigation of algal bloom in a lake, J. Biol. Phys. 42(1) (2016) 147-165.

[9] P.K. Tiwari, A.K. Misra, E. Venturino, The role of algae in agriculture: A mathematical study, J. Biol. Phys. 43(2) (2017) 297-314.

[10] S. Chakraborty et al., Effects of fertilizers used in agricultural fields on algal blooms, Eur. Phys. J. Special Topics 226 (2017) 2119-2133.

[11] R.J. Diaz, R. Rosenberg, Spreading dead zones and consequences for marine ecosystems, Science 321 (2008) 926-929.

[12] X. Gao, J.C. Ren, Z.X. Zong, Studies on the nutrient energetics of Microcytis aeruginosa, Acta Scientiarum Naturalium 30(4) (1994) 461-469.

[13] N.N. Rabalais et al., Dynamics and distribution of natural and human-caused hypoxia, Biogeosciences 7 (2010) 585-619.

[14] W. Carmichael, Cyanobacterial harmful algal blooms: state of the science and research needs, Springer (2008).

[15] Interview: Raising awareness about global toxic algae bloom. http://www.digitaljournal.com/news/environment/interview-raising-awareness-about-global-toxic-a

[16] Water quality standards. https://www.pca.state.mn.us/water/water-quality-standards.

[17] H. Sarmento, J.M. Gasol, Use of phytoplankton-derived dissolved organic carbon by different types of bacterioplankton, Environ. Microbiol. 14 (2012) 2348-2360.

[18] Y. Aota, H. Nakajima, Mutualistic relationships between phytoplankton and bacteria caused by carbon excretion from phytoplankton, Ecol. Res. 16 (2001) 289-299.

[19] L. Riemann, G.F. Steward, F. Azam, Dynamics of bacterial community composition and activity during a mesocosm diatom bloom, Appl. Environ. Microbiol. 66 (2000) 578-587.

[20] M.R. Seyedsayamdost et al., The Jekyll and Hyde chemistry of Phaeobacter gallaciensis, Nature Chem. 3 (2011) 331-335.

[21] A.M. Amaro et al., Identification and characterization of potentially algal-lytic marine bacteria strongly associated with the toxic dinoflagellate Alexandium catenella, J. Eukaryot. Microbiol. 52 (2005) 191-200.

[22] S.A. Amin et al. Interaction and signalling between a cosmopolitan phytoplankton and associated bacteria, Nature 522 (2015) 98-101. 
[23] M. Landa et al., Shifts in bacterial community composition associated with increased carbon cycling in a mosaic of phytoplankton blooms, ISME J. 10 (2015) 39-50.

[24] L. Philippot et al., Going back to the roots: the microbial ecology of the rhizosphere. Nat. Rev. Microbiol. 11 (2013) 789-799.

[25] M.T. Croft et al., Algae acquire vitamin B12 through a symbiotic relationship with bacteria, Nature 438 (2005) 90-93.

[26] M. Teplitski, S. Rajamani, Signal and nutrient exchange in the interactions between soil algae and bacteria, In biocommunication in soil microorganisms; Witzany, G., Ed.; Springer: Berlin, Germany (2011) 413-426.

[27] L.E. Gonzalez, Y. Bashan, Increased growth of the microalga Chlorella vulgaris when coimmobilized and co-cultured in alginate beads with the plant-growth-promoting bacterium Azospirillum brasilense, Appl. Environ. Microbiol. 66 (2000) 1527-1531.

[28] B.H. Kim et al., Role of Rhizobium, a plant growth promoting bacterium, in enhancing algal biomass through mutualistic interaction, Biomass Bioenerg. 69 (2014) 95-105.

[29] R. Ramanan et al., Phycosphere bacterial diversity in green algae reveals an apparent similarity across habitats, Algal Res. 8 (2015) 140-144.

[30] G.J. Doucette, Interactions between bacteria and harmful algae: a review, Nat. Toxins 3(2) (1995) 65-74.

[31] K. Gopalsamy, Stability and oscillations in delay differential equations of population dynamics, Mathematics and its applications. Kluwer Academic Pub. Dordrecht 74 (1992).

[32] S. Ruan, J. Wei, Stability and bifurcation in a neural network model with two delays, Physica D 130 (1999) 255-272.

[33] S. Ruan, J. Wei, On the zeros of transcendental functions with applications to stability of delay differential equations with two delays, Dyn. Contin. Disc. Impul. Syst. Ser. A Math. Anal. 10 (2003) $863-874$.

[34] T. Amemiya et al., Stability and dynamical behavior in a lake-model and implications for regime shifts in real lakes, Ecol. Model. 206 (2007) 54-62.

[35] A.K. Misra, Modeling the depletion of dissolved oxygen due to algal bloom in a lake by taking Holling type-III interaction, Appl. Math. Comput. 217, (2011) 8367-8376.

[36] R.H. Whittaker, Communities and Ecosystems. Macmillan, New York (1975).

[37] S. Ruan, Oscillations in plankton models with nutrient recycling, J. Theor. Biol. 208 (2001) 15-26.

[38] M. Gao, H. Shi, Z. Li, A planktonic resourceconsumer model with a temporal delay in nutrient recycling, J. Math. Anal. Appl. 339 (2008) 511-516.

[39] K. Das, S. Ray, Effect of delay on nutrient cycling in phytoplankton-zooplankton interactions in estuarine system, Ecol. Model. 215 (2008) 69-76.

[40] A.K. Misra, P. Chandra, V. Raghavendra, Modeling the depletion of dissolved oxygen in a lake due to algal bloom: Effect of time delay, Adv. Water Res. 34 (2011) 1232-1238. 
[41] A.K. Misra, P.K. Tiwari, P. Chandra, Modeling the control of algal bloom in a lake by applying some external efforts with time delay, Differ. Equ. Dyn. Syst. DOI 10.1007/s12591-017-0383-5 (2017).

[42] R. Diaz, N.N. Rabalais, D.L. Breitburg, Agriculture's impact on aquaculture: hypoxia and eutrophication in marine waters, https://www.oecd.org/tad/sustainable-agriculture/49841630.pdf, (2012).

[43] Y. Li, Effect of N, P concentration on growth rate, Acta Ecologica Sinica 26 (2006) 317-325.

[44] J.L. Mouget et al., Algal growth enhancement by bacteria: is consumption of photosynthetic oxygen involved?. FEMS Microbiology Ecology, 18(1) (1995) 35-44.

[45] K.C. Marshall, Cyanobacterial-heterotrophic bacterial interactions. In: Microbials MatsPhysiological Ecology of Benthic Microbial Communities (Y. Cohen, E. Rosenberg Eds.), American Society for Microbiology, Washington DC. (1989) 239-245.

[46] R. Ukeles, J. Bishop, Enhancement of phytoplankton growth by marine bacteria, J. Phycol. 11 (1975) 142-149.

[47] A.K. Jones, The interaction of algae and bacteria. In: Microbial Interactions and Communities (Bull, A.T. and Slater, J.H., Eds.), Academic Press, New York, (1982) 189-247.

[48] E. Beretta, Y. Kuang, Modeling and analysis of a marine bacteriophage infection, Math. Biosci. 149 (1998) 57-76.

[49] A.K. Misra, P. Chandra, J.B. Shukla, Mathematical modeling and analysis of the depletion of dissolved oxygen in water bodies. Nonlinear Anal. RWA. 7 (2006) 980-996.

[50] J.B. Shukla, A.K. Misra, P. Chandra, Mathematical modeling of the survival of a biological species in polluted water bodies, Diff. Equ. Dyn. Syst. 15(3/4) (2007) 209-230.

[51] P. Hohener, O. Atteia, Multidimensional analytical models for isotope ratios in groundwater pollutant plumes of organic contaminants undergoing different biodegradation kinetics, Adv. Water. Resour. 33 (2010) 740-751.

[52] P.K. Tiwari et al., Modeling the direct and indirect effect of pollutants on the fish survival in water bodies, J. Biol. Syst. 25(03) (2017) 521-543.

[53] P.K. Tiwari et al., Human population effects on the Ulsoor lake fish survival, J. Biol. Syst. 26(04) (2018) 603-632.

[54] S. Rinaldi et al., Modeling and control of river quality, McGraw-Hill Inc., U.K. (1979).

[55] H.I. Freedman, J.W.H. So, Global stability and persistence of simple food chains, Math. Biosci. 76 (1985) 69-86.

[56] C. Castillo-Chavez, B. Song, Dynamical models of tuberculosis and their applications, Math. Biosci. Eng. 1 (2004) 361-404.

[57] X. Wang, A simple proof of Descartes's rule of signs, Am. Math. Mon. 111(6) (2004) 525-526.

[58] A. Huppert, B. Blasius, L. Stone, A model of phytoplankton blooms. Am. Nat. 159(2) (2002) 156-171. 
[59] T. Park, A matlab version of the Lyapunov exponent estimation algorithm of wolf et al. https ://www . mathworks. com/matlabcentral/fileexchange/48084-lyapunov-exponent-estimation-from-a-

[60] A. Wolf et al., Determining Lyapunov exponents from a time series, Physica D Nonlinear Phenomena 16 (1985) 285-317.

[61] S.M. Blower, H. Dowlatabadi, Sensitivity and uncertainty analysis of complex models of disease transmission: an HIV model, as an example, Int. Stat. Rev. 62 (1994) 229-243.

[62] S. Marino et al., A methodology for performing global uncertainty and sensitivity analysis in systems biology, J. Theor. Biol. 254(1) (2008) 178-196.

[63] Climate Change and Harmful Algal Blooms. https : //www .epa.gov/nutrientpollution/climate-change-and-ha

[64] J. Zhou et al., A review of the relationship between algae and bacteria in harmful algal blooms, Acta Ecol. Sin. 34 (2014) 269-281.

[65] J.L. Fuentes et al., Impact of microalgae-bacteria interactions on the production of algal biomass and associated compounds, Marine drugs 14(5) (2016) 100.

[66] H. Malchow, S. Petrovskii, E. Venturino, Spatiotemporal patterns in Ecology and Epidemiology, CRC, 2008.

[67] S. Chakraborty et al., Spatial dynamics of a nutrient-phytoplankton system with toxic effect on phytoplankton, Math. Biosci. 264 (2015) 94-100.

[68] P.K. Tiwari et al., Effect of cross-diffusion on the patterns of algal bloom in a lake: A nonlinear analysis, Nonlinear Studies, 21(3) (2014) 443-462. 

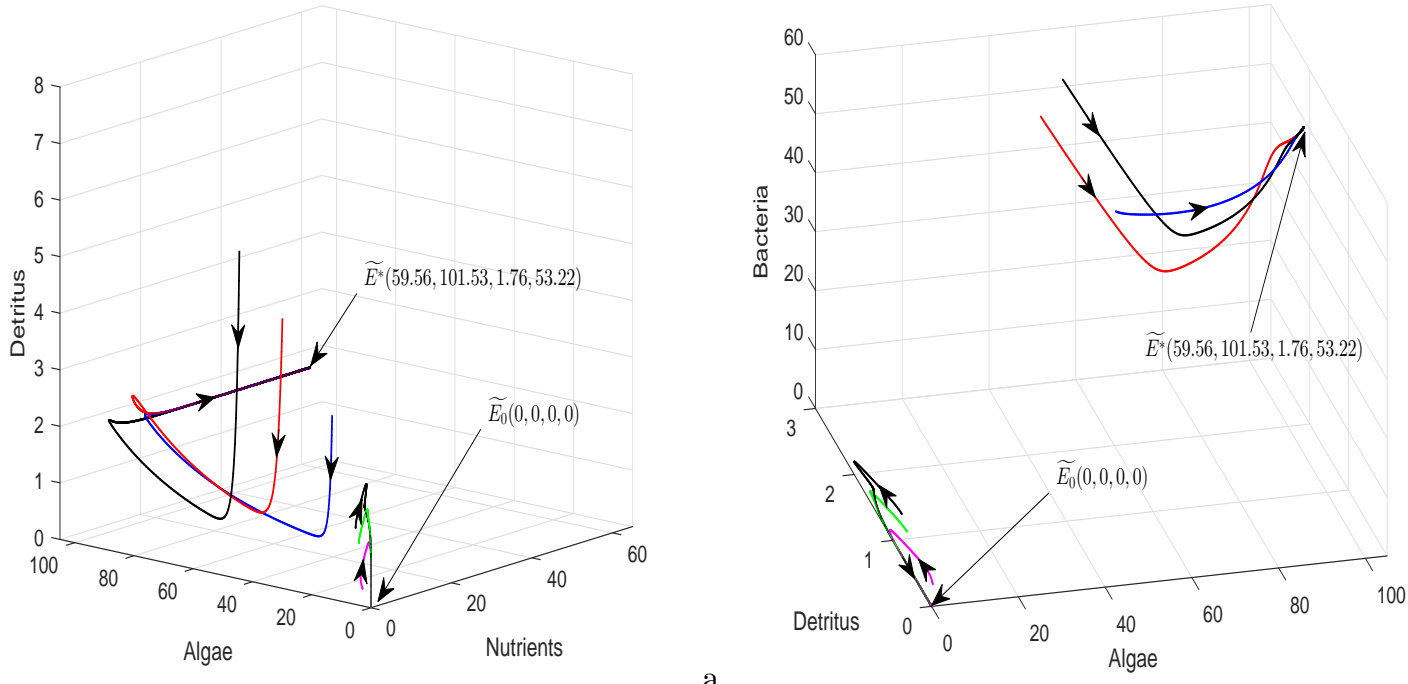

Figure 2: Figure shows bi-stability behavior of the system (5.1). The trajectories with different initial conditions lead to different attractors. Parameters values are same as in Table 1 except $\theta_{1}=15.5, \alpha_{1}=0.3, \pi_{1}=0.23$ and $\lambda_{1}=0.26$. 

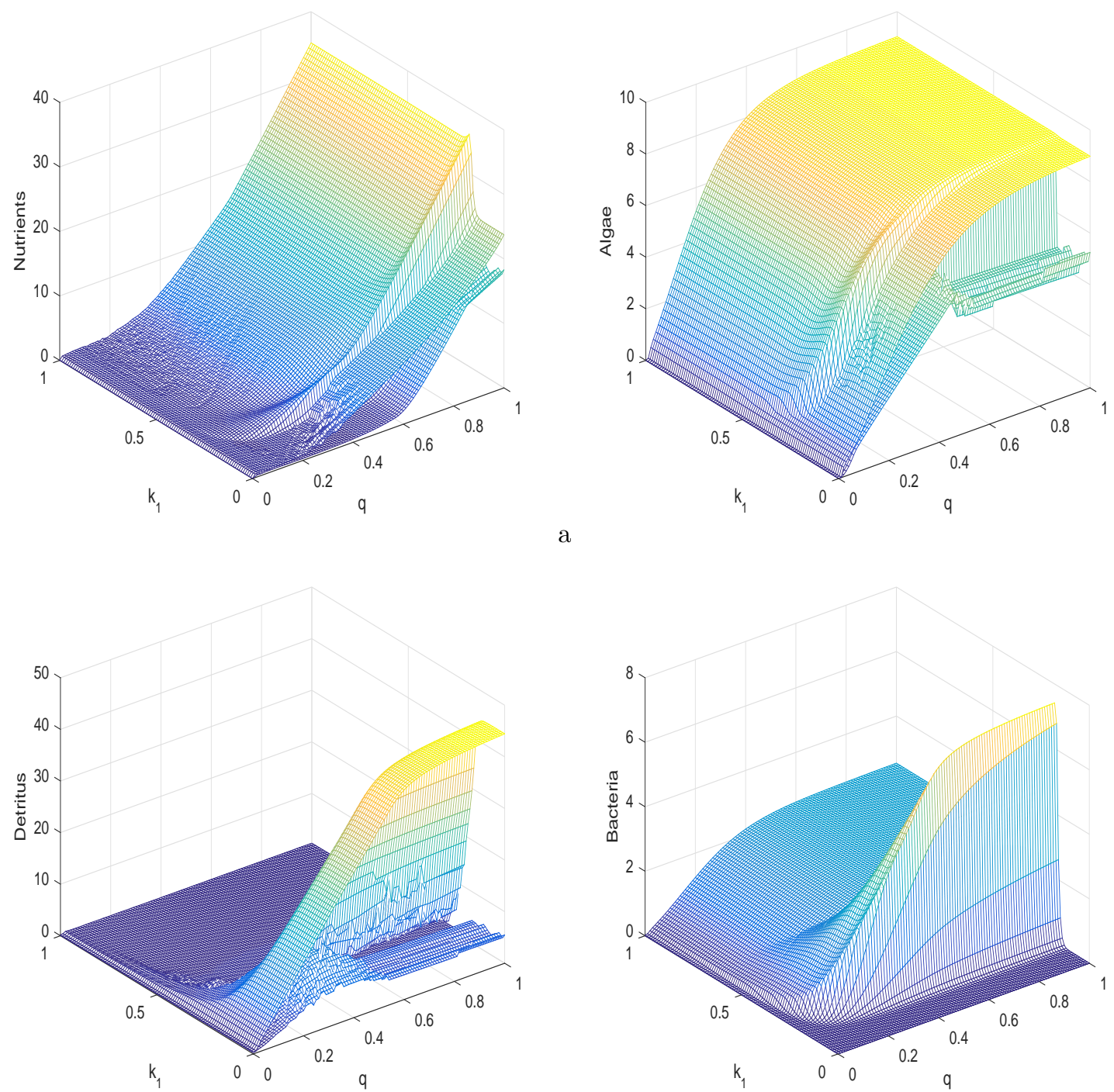

Figure 3: The equilibrium values of nutrients, algae, detritus and bacteria as functions of $q$ and $k_{1}$ with initial conditions $(0.12,1.15,0.1,0.06)$. Rest of the parameters values are same as in Table 1. 

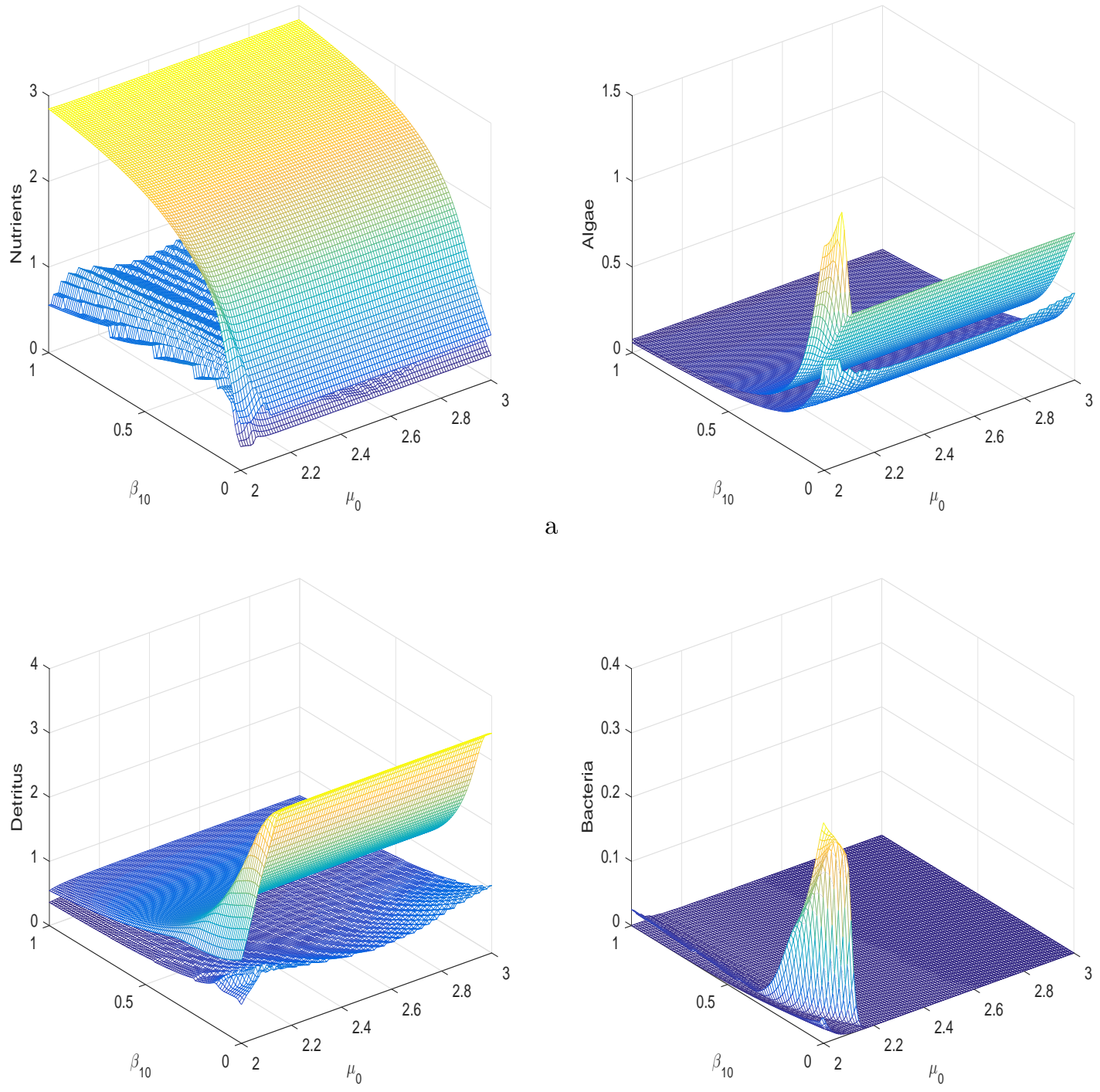

Figure 4: The equilibrium values of nutrients, algae, detritus and bacteria as functions of $\mu_{0}$ and $\beta_{10}$ with initial conditions $(0.12,1.15,0.1,0.06)$. Rest of the parameters values are same as in Table 1. 

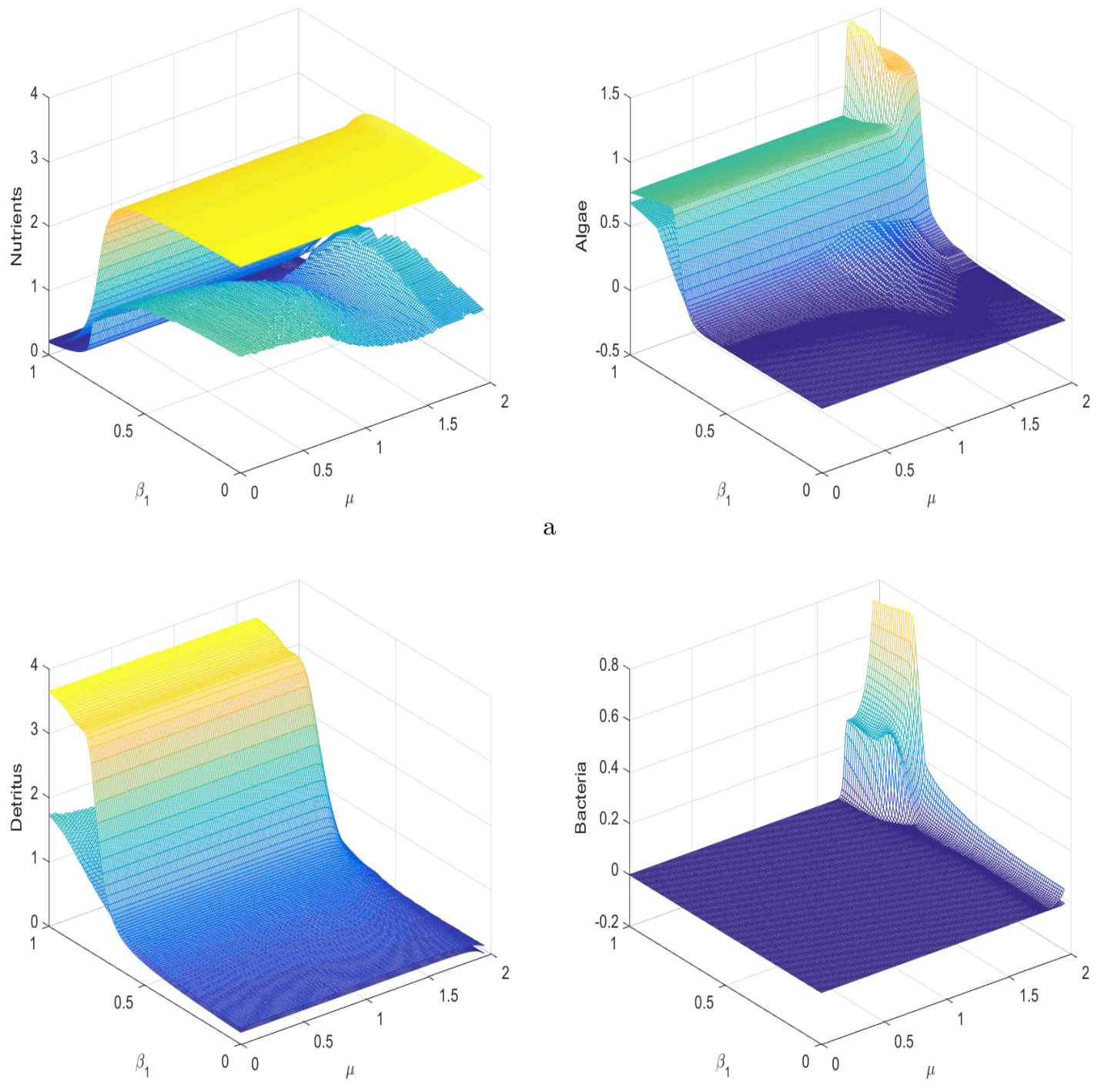

Figure 5: The equilibrium values of nutrients, algae, detritus and bacteria as functions of $\mu$ and $\beta_{1}$ with initial conditions $(0.12,1.15,0.1,0.06)$. Rest of the parameters values are same as in Table 1. 

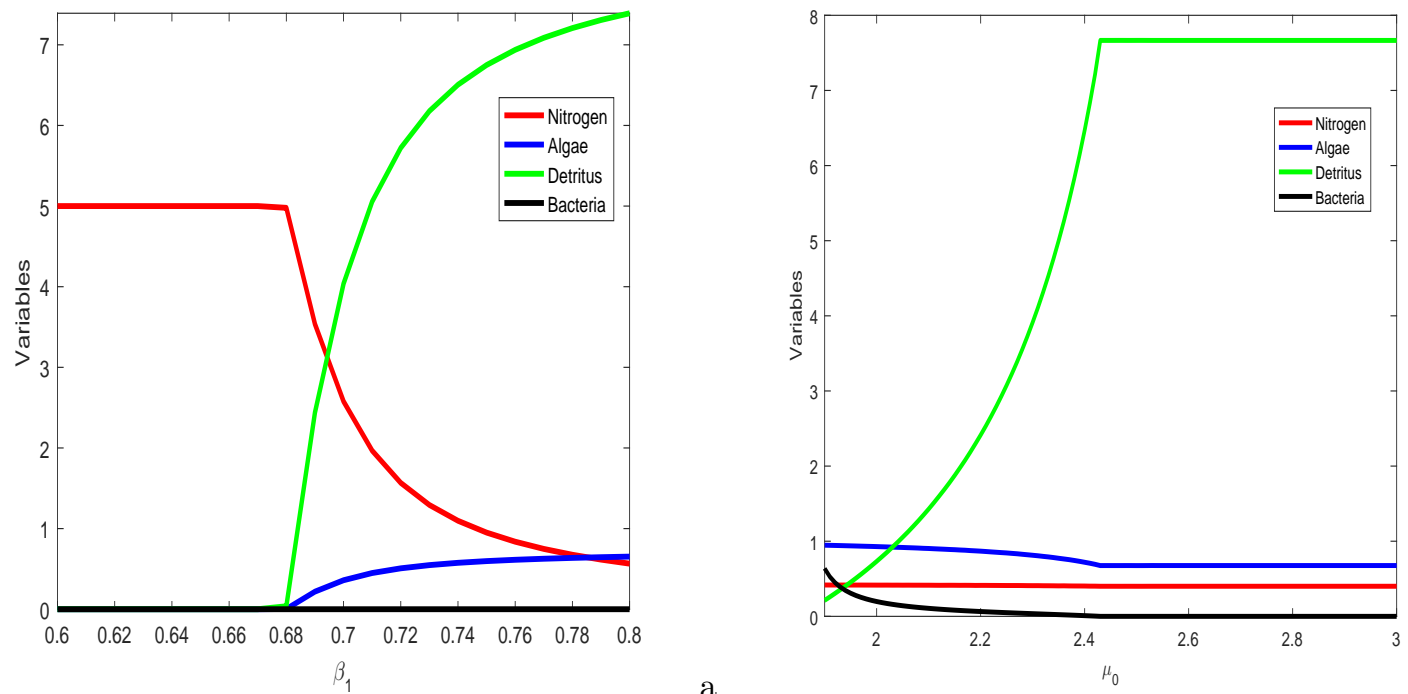

$\mathrm{b}$

Figure 6: Transcritical bifurcations between equilibria (a) $E_{0}$ and $E_{1}$, left to right, when $\mu=0.8$ and (b) $E^{*}$ and $E_{1}$, left to right, when $\mu=1.85$ and the other parameters values are same as in Table 1 . Initial conditions are chosen as $(0.12,1.15,0.1,0.06)$. 

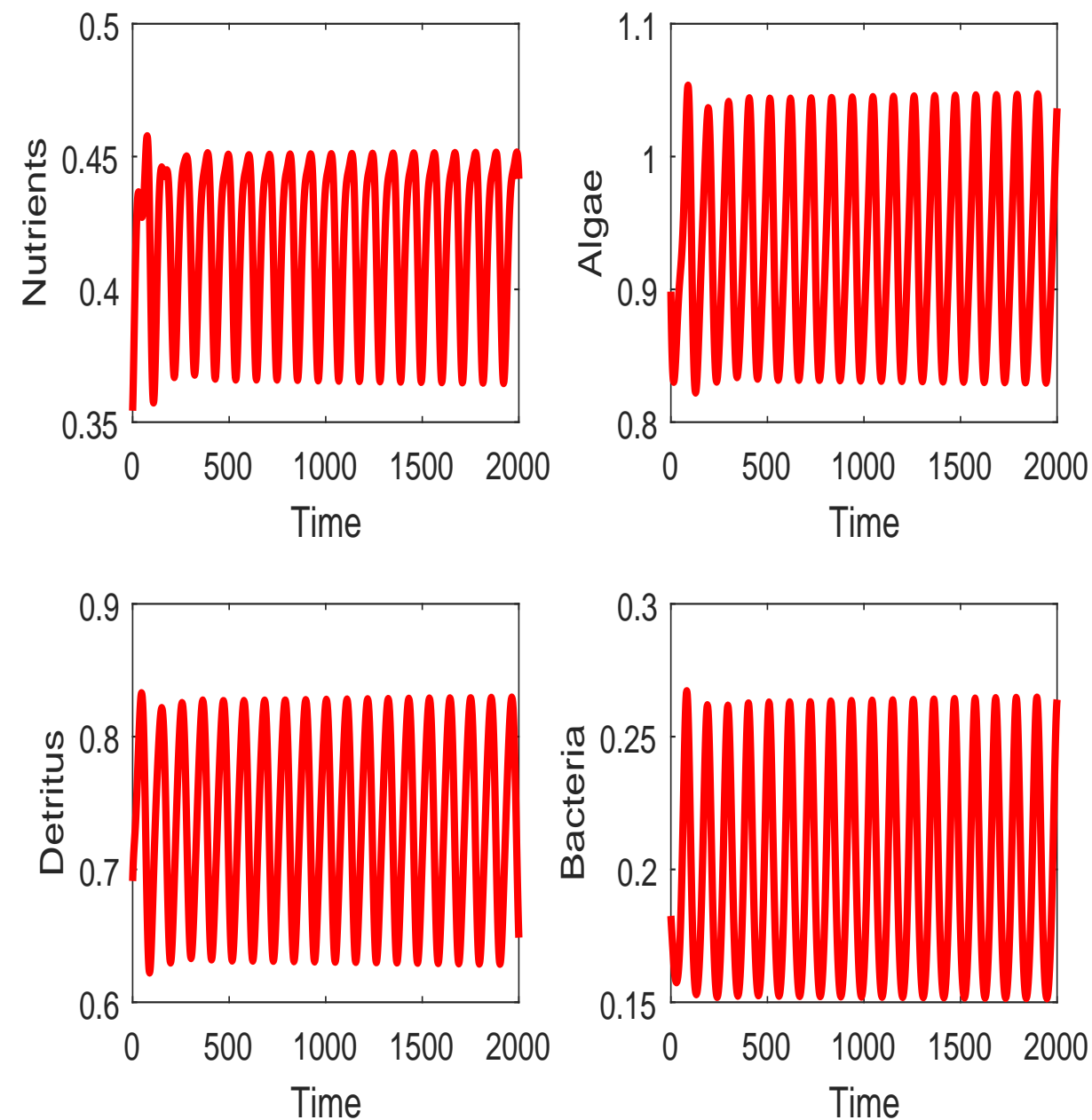

Figure 7: System (8.1) shows limit cycle oscillation for $\tau=15.2$ days. Initial conditions are chosen as $(0.3543,0.8983,0.6913,0.1825)$. Parameters values are same as in Table 1. 

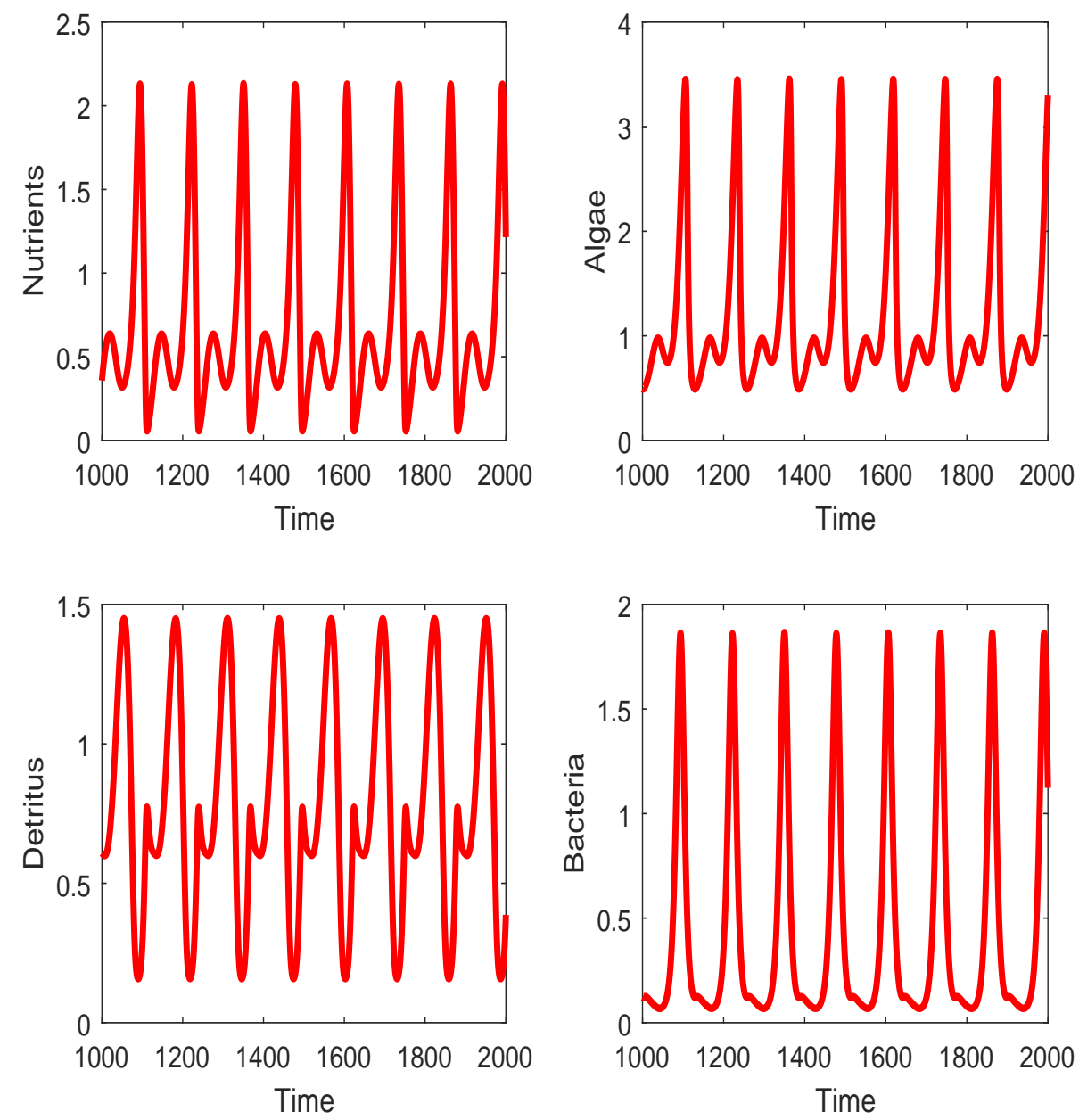

Figure 8: System (8.1) shows 2-periodic solutions for $\tau=20$ days. Initial conditions are chosen as $(0.3543,0.8983,0.6913,0.1825)$. Parameters values are same as in Table 1. 

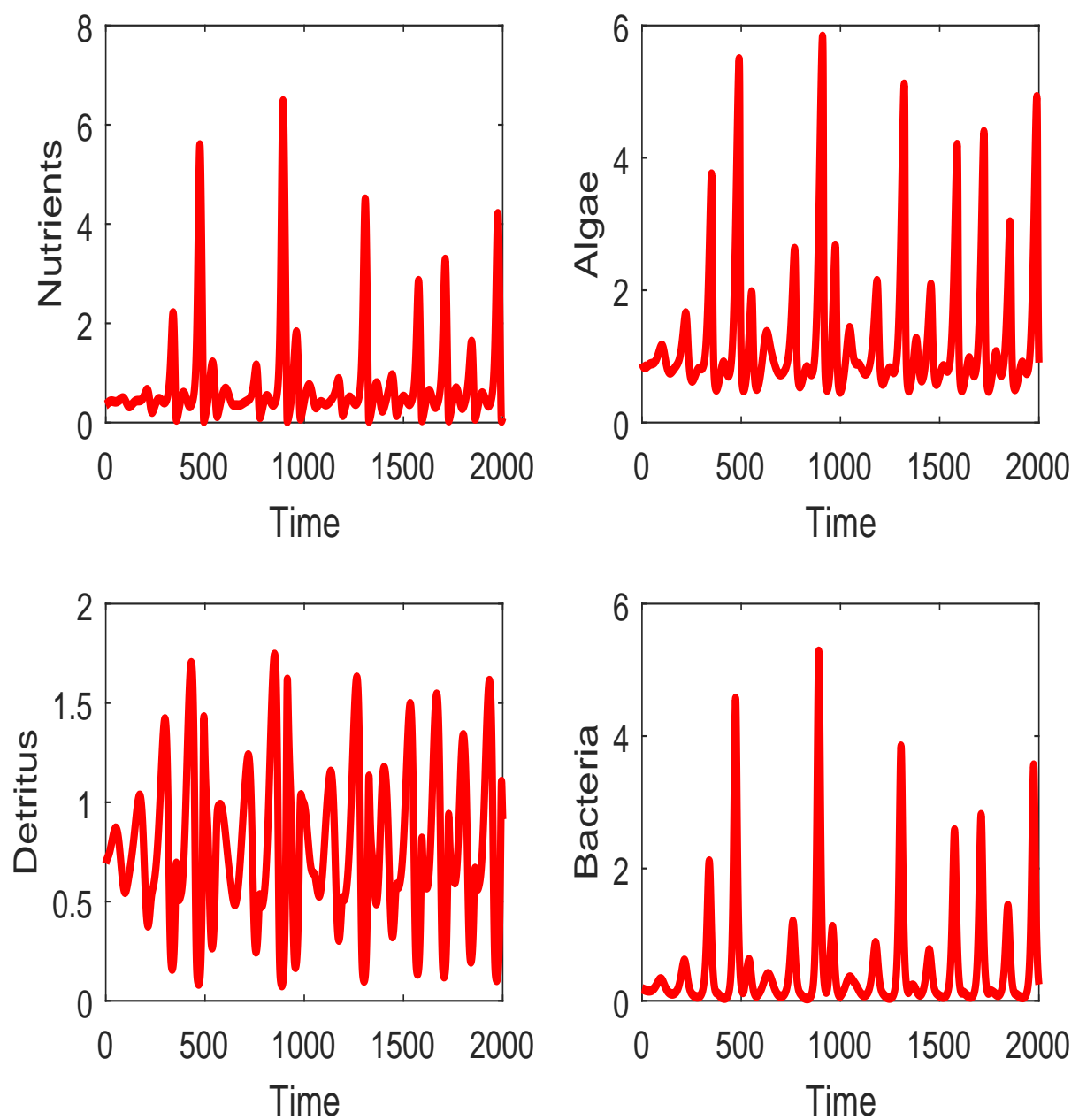

Figure 9: System (8.1) shows chaotic oscillations for $\tau=21.5$ days. Initial conditions are chosen as $(0.3543,0.8983,0.6913,0.1825)$. Parameters values are same as in Table 1. 

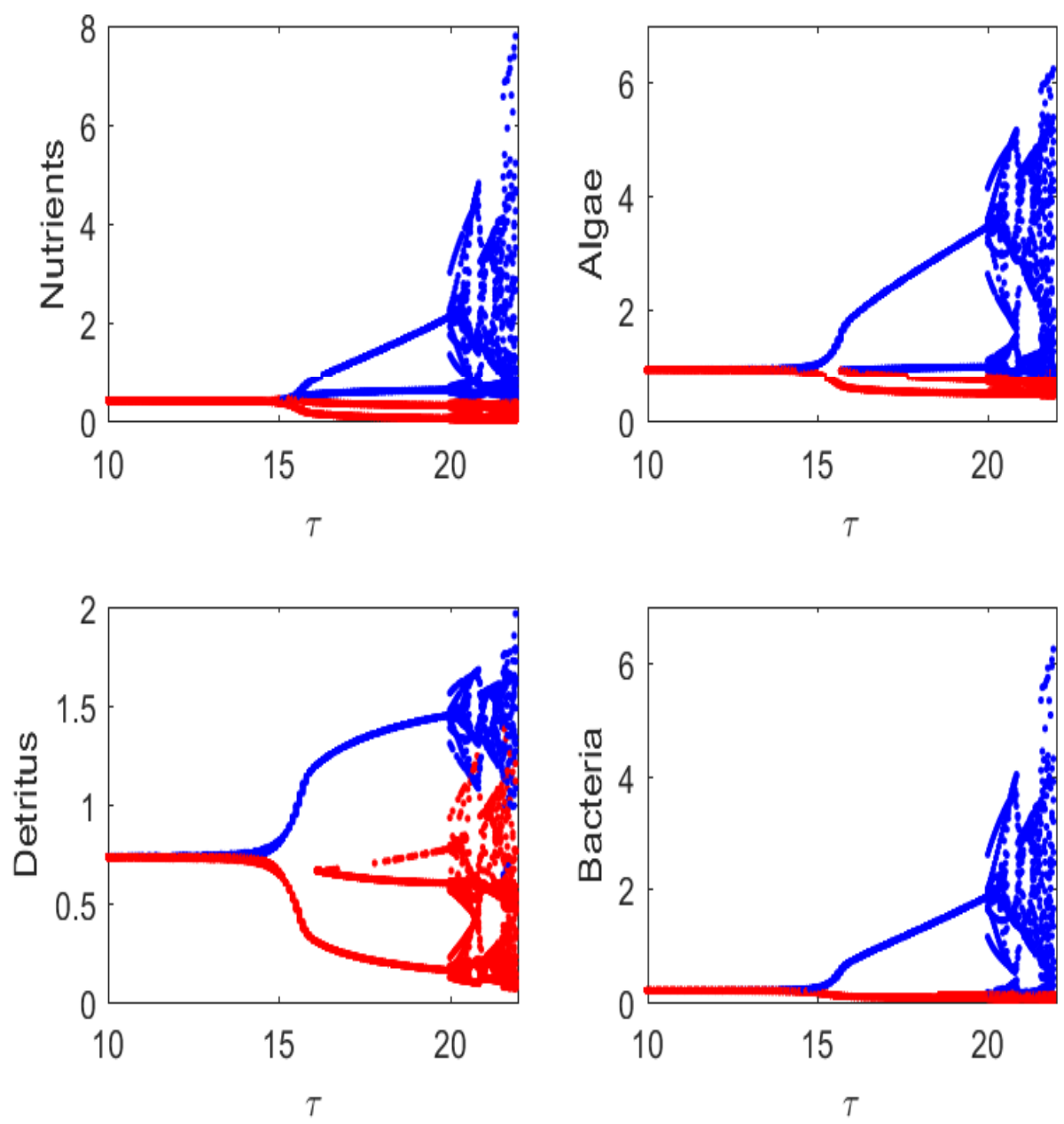

Figure 10: Bifurcation diagram of the system (8.1) with respect to $\tau \in[10,22]$. Here, the maximum and minimum values of the oscillations are plotted in blue and red colors, respectively. Initial conditions are chosen as $(0.3543,0.8983,0.6913,0.1825)$. Parameters values are same as in Table 1. 


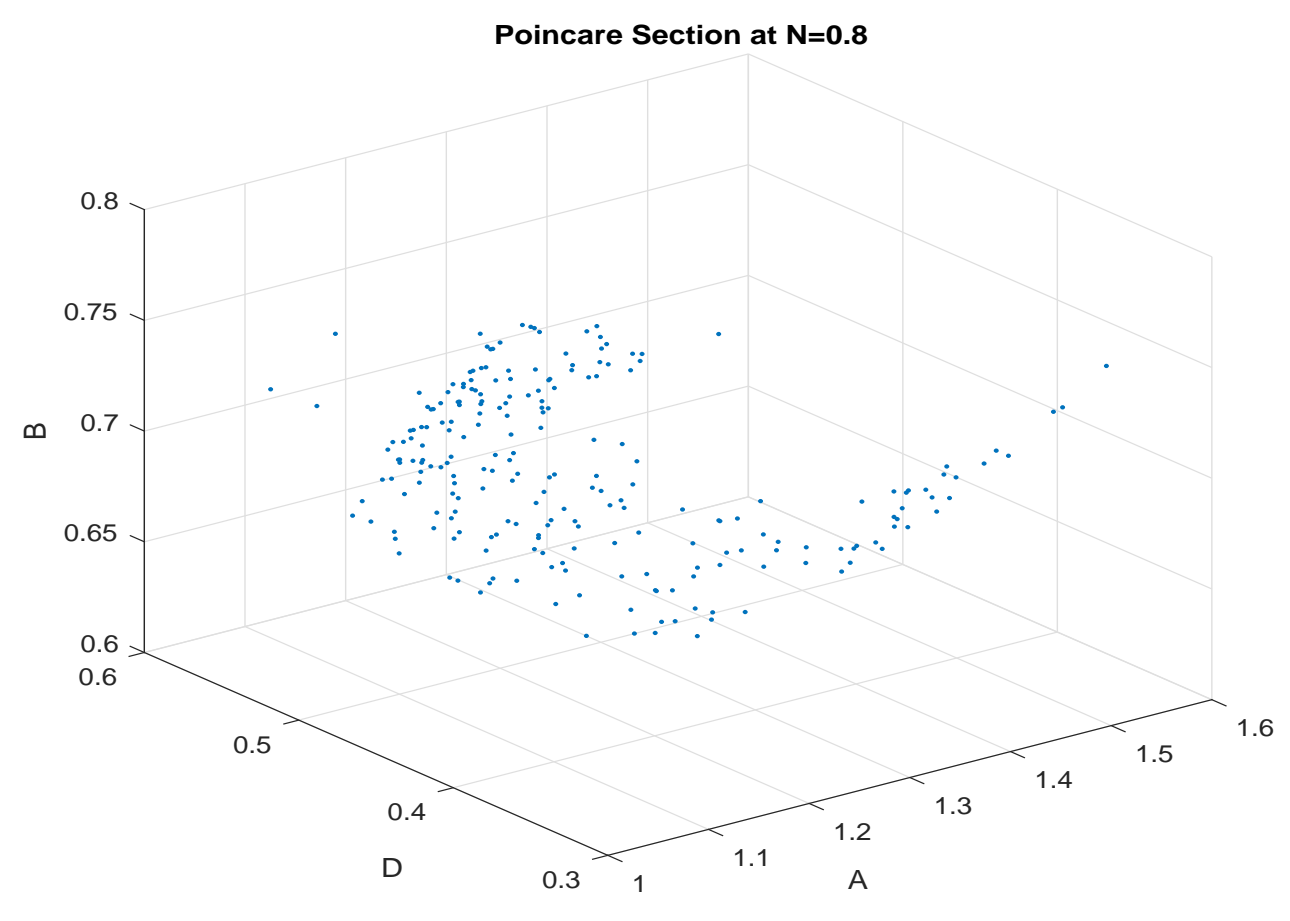

Figure 11: Poincare map of the system (8.1) in the $A-D-B$ space $(N=10)$ for $\tau=21.5$ days. Initial conditions are chosen as $(0.3543,0.8983,0.6913,0.1825)$. Parameters values are same as in Table 1 . 


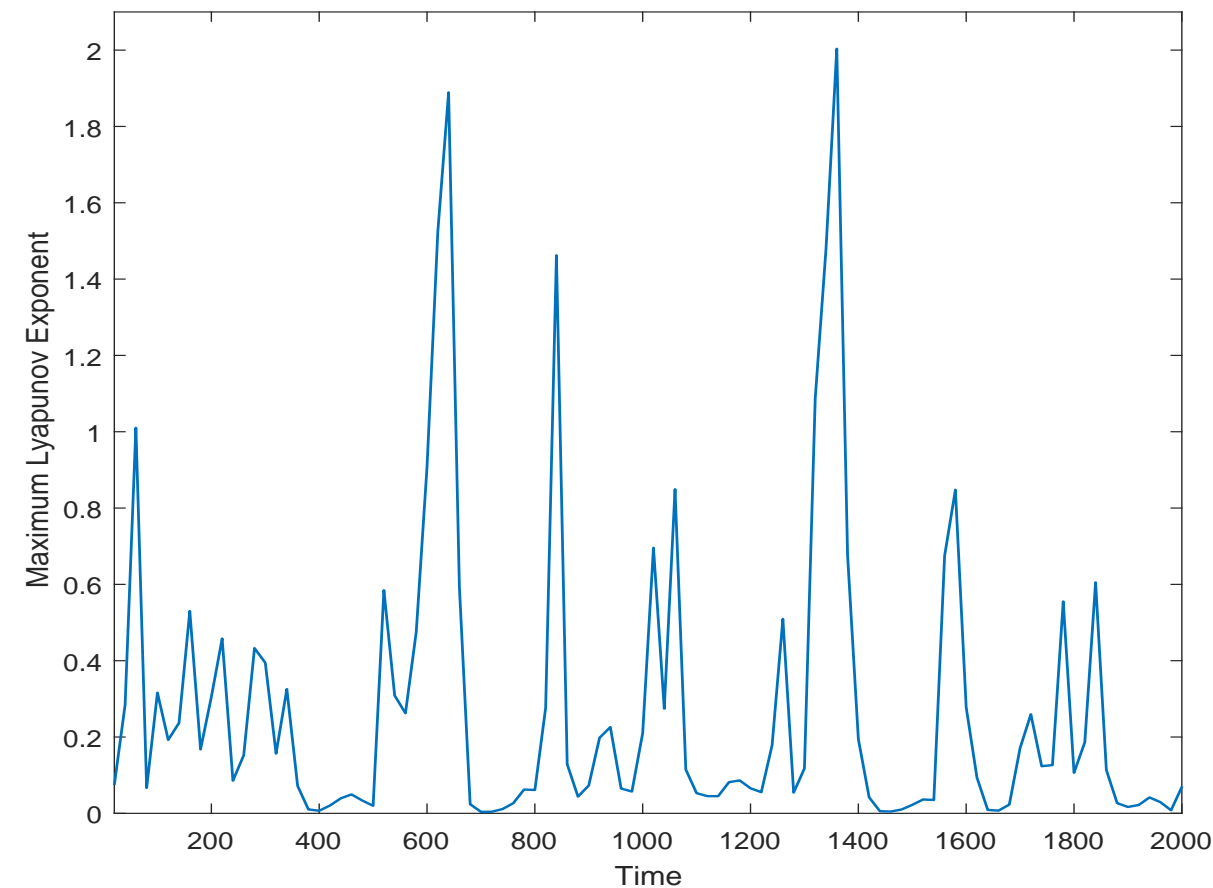

Figure 12: The maximum Lyapunov exponent of the system (8.1) for $\tau=21.5$ days. Initial conditions are chosen as $(0.3543,0.8983,0.6913,0.1825)$. Parameters values are same as in Table 1. 

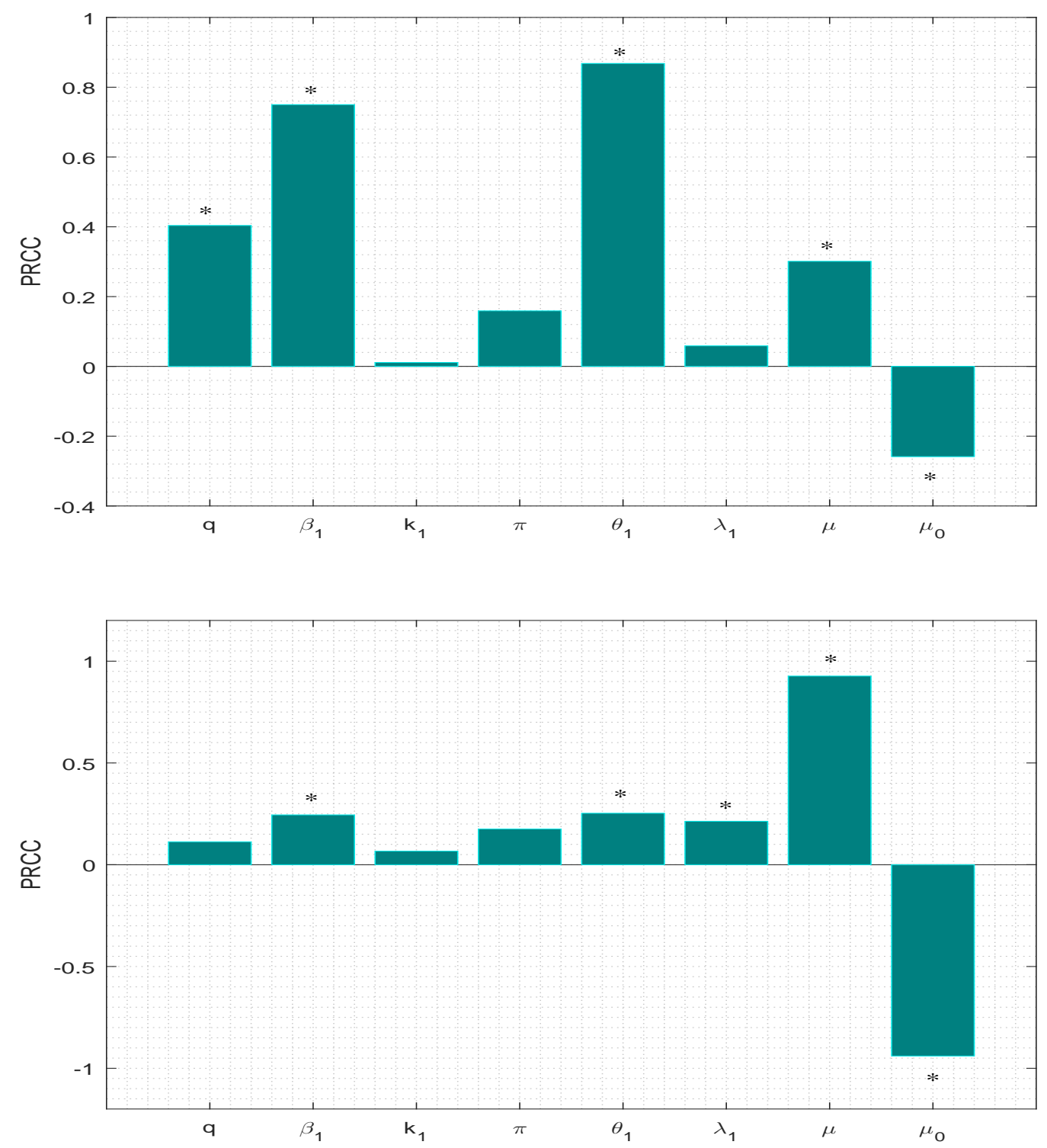

Figure 13: Effect of uncertainty of the model (2.1) on (a) algae and (b) bacteria. Significant parameters are marked by *. Initial conditions are chosen as $(0.12,1.15,0.1,0.06)$. The mean values of parameters are chosen as in Table 1. 